\title{
Hydrophytic Cyperaceae from the Araguaia river basin, Brazil ${ }^{1}$
} Cyperaceae hidrófilas da bacia do rio Araguaia, Brasil

\author{
Adriana Luiza Ribeiro de Oliveira ${ }^{2,4}$, André dos Santos Bragança Gil ${ }^{3}$ \& Claudia Petean Bove ${ }^{2}$
}

\begin{abstract}
Cyperaceae is a cosmopolitan family with over 5,000 species, which are often found in wet habitats. In this study we found 31 hydrophylic species of Cyperaceae, belonging to 10 genera (Ascolepis, Calyptrocarya, Cyperus, Eleocharis, Fimbristylis, Fuirena, Lipocarpha, Pycreus, Rhynchospora and Scleria), that occur in the Araguaia River Basin, located mainly within the Brazilian Cerrado (savanna). Short descriptions, geographic distribution, illustrations, identification keys and comments are presented.
\end{abstract}

Key words: cerrado, hydrophytic plants, wetlands.

\section{Resumo}

Cyperaceae é uma família cosmopolita com mais de 5.000 espécies que ocorrem frequentemente em áreas úmidas. Foram encontradas 31 espécies desta família pertencentes a 10 gêneros (Ascolepis, Calyptrocarya, Cyperus, Eleocharis, Fimbristylis, Fuirena, Lipocarpha, Pycreus, Rhynchospora e Scleria) na bacia hidrográfica do Rio Araguaia, que está em sua maior parte inserida no Cerrado Brasileiro. São apresentadas breves descrições, distribuição geográfica, ilustrações, chaves de identificação e comentários.

Palavras-chave: cerrado, plantas hidrófilas, áreas úmidas.

\section{Introduction}

The Araguaia River, a tributary of the Tocantins River, covers $373,000 \mathrm{~km}^{2}$ (Lima et al. 2004) and lies within the Brazilian states of Goiás, Mato Grosso, Tocantins, and Pará (Fig. 1). Most of the Araguaia River Basin is located within the Brazilian Cerrado (tropical savanna), which is ranked among the 25 world biodiversity hotspots (Myers et al. 2000). In the area there is a predominance of plinthosoils which are poorly drained and are usually found in low, flat areas prone to flooding (Resende et al. 1995), forming temporary or permanent swamps with high plant diversity.

Cyperaceae Juss. is a cosmopolitan family comprising species that often dominate wetland ecosystems. According to Goetghebeur (1998) and Govaerts et al. (2007), this family is composed of approximately 5,000 species that occur on all continents except Antarctica, and that have their highest diversity in Africa and the Neotropics. Alves et al. (2010) recorded 594 species from 40 genera in Brazil. The present study aims to make the first taxonomic essay of the Cyperaceae focused on the Araguaia River Basin, in order to provide identification keys, taxonomic descriptions and illustrations of this family in the region.

\section{Material and Methods}

The term hydrophytic, according to Cook (1996), means plants 'whose photosynthetically active parts are permanently or, at least, for several months each year submerged in water or float on the surface of water'. Life forms categories (amphibious, emergent and submerged-fixed) follow Pedralli (1988) and were defined according to field data and literature (Alves et al. 2009). The

\footnotetext{
${ }^{1}$ Part of the first author's Master's dissertation.

${ }^{2}$ Universidade Federal do Rio de Janeiro - UFRJ, Programa de Pós Graduação em Ciências Biológicas - Botânica, Depto. Botânica, Museu Nacional, 20940-040, Rio de Janeiro, Brazil.

${ }^{3}$ Faculdade de Tecnologia do Estado de São Paulo (FATEC/CB), Centro Paula Souza, Pça. Cel. Fernando Prestes 30, 01124-060, São Paulo, SP, Brazil.

${ }^{4}$ Corresponding author: ribeirosenna1@gmail.com
} 
material studied came from collections carried out between $5-20^{\circ} \mathrm{S}$ and $45-55^{\circ} \mathrm{W}$, a region that coincides approximately with the southern limit of Alto Araguaia and the northern limit of Formoso do Araguaia (Fig. 1). Sampling expeditions were carried out mainly along state roads (MT-100, MT126, MT-326, GO-164, GO-173, GO-221, GO-324, GO-334 and GO-530) and federal roads (BR-070, BR-158 and BR-251) in both rainy and dry seasons (1997, 1999, 2004, 2006 and 2007). Surveyed habitats included seasonally and permanently flooded areas. Voucher material was deposited in the herbarium of Museu Nacional do Rio de Janeiro - UFRJ (R). Additional material for the study was obtained from the herbaria R, RB, UB and UFG (acronymous according Thiers 2010). The taxa treated here followed Alves et al. (2010). Measures of achene length did not include the tubercle. Geographic distribution followed Goetghebeur (1998) and Adams (1994).

\section{Results}

In the Araguaia River Basin there are 31 species of hydrophytic Cyperaceae from ten genera. Eleocharis (10 spp.) and Rhynchospora (19 spp.) are the most representative.

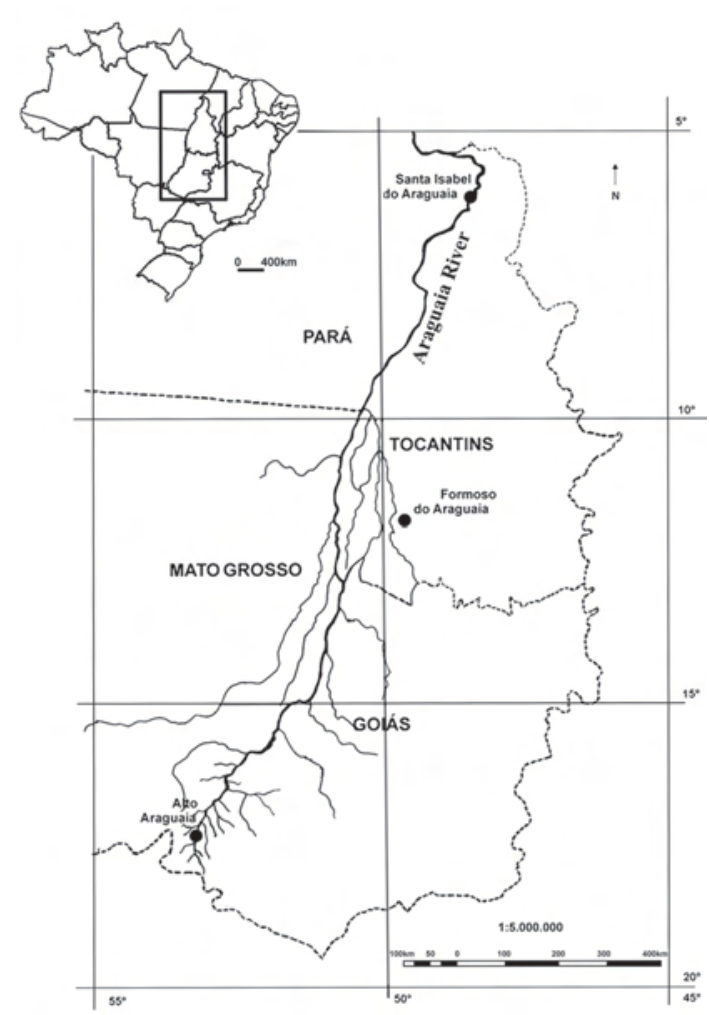

Figure 1 - Map of the Araguaia River Basin.

\section{Key to the genera of hydrophytic Cyperaceae of the Araguaia River Basin}

1. Spikelets unisexual or bisexual and staminate.

2. Spikelets bisexual and staminate

9. Rhynchospora

2'. Spikelets unisexual.

3. Achenes enclosed in utricles

2. Calyptrocarya

3'. Achenes not enclosed in utricles 10. Scleria

1'. Spikelets only bisexual.

4. Leaves reduced to sheaths, bracts absent

4. Eleocharis

4'. Leaves with sheath and blade, bracts present.

5. Spike.

6. Achenes enclosed in utricles, glumes and prophylls absent

1. Ascolepis

6'. Achenes not enclosed in utricles, glumes and prophylls present

7. Lipocarpha

5. Anthela.

7. Glumes distichous

8. Styles 3-fid, achenes trigonous

3. Cyperus

8'. Styles 2-fid, achenes biconvex

8. Pycreus

7’. Glumes spiral.

9. Spikelets single, perianth absent

5. Fimbristylis

9'. Spikelets fasciculate, perianth present

6. Fuirena 
1. Ascolepis Nees ex Steud., Syn. Pl. Glumec. 2: 105.1855

1.1 Ascolepis brasiliensis (Kunth) Benth. ex C.B. Clarke in Durand \& Schinz, Consp. Fl. Afr. 5: 651. 1895.

Fig. 2a-d

Plants emergent, $20-80 \mathrm{~cm}$ tall. Leaves $2-23 \times$ $0.1-0.3 \mathrm{~cm}$, linear, apex obtuse, adaxial surface pilose. Spikes $0.5-1 \times 0.5-0.7 \mathrm{~cm}$, multiflorous, sessile, conical; bracteoles $0.3-4 \times 0.1-0.3 \mathrm{~mm}$, oblong or oblong-oval, apex acuminate or apiculate, flat, glabrous, 1-nerved, membranous; utricles $2-3 \times 1.5-2 \mathrm{~mm}$, oval, apex acuminate, white, winged, paleaceous, glabrous. Achenes 1.6-1.8 $\times 0.4-0.5 \mathrm{~mm}$, oblong or elliptic, brown. Material examined: GOIÁS: Britânia, 12.XI.1999, fl. and fr., C.P. Bove et al. 548 (R). Palestina, 11.IV.2006, fl., C.P. Bove et al. $1640 b$ (R). Niquelândia, 25.I.1972, H.S. Irwin et al. 35041 (UB).

Ascolepis brasiliensis is similar to Lipocarpha humboldtiana Nees. The former has a winged utricle and oblong bracteoles, whereas $L$. humboldtiana has no utricle and oval-triangular bracteoles. A. brasiliensis is found in lakes and swamps with buriti palms (Mauritia flexuosa L.f.), locally known as buritizais.
It occurs from Panama to Argentina, as well as in tropical Africa and Madagascar.

2. Calyptrocarya Nees, Linnaea 9(3): 304. 1834. 2.1 Calyptrocarya glomerulata (Brongn.) Urb., Symb. Antill. 2: 169. 1900.

Fig. 2e-f

Plants emergent, $20-50 \mathrm{~cm}$ tall. Leaves 12.5 $20 \times 0.4-0.5 \mathrm{~cm}$, linear, linear-lanceolate, apex acute, margins glabrous or scabrous. Anthela 3-4 lateral, $0.7-2 \times 0.9-2 \mathrm{~mm}$. Spikelets $2-3 \times 2-3 \mathrm{~mm}$; glumes of similar staminate and pistillate spikelets, $1-1.5 \times$ $0.5-1.2 \mathrm{~mm}$, oval to widely oval, apex acute; utricles brown, membranous, pubescent. Achenes 1-1.5× $1 \mathrm{~mm}$, ovoid to ellipsoid, white, stipitate.

Material examined: GOIÁS: Caiapônia, Cachoeira Abóbora, 18.XI.1999, fr., C.P. Bove et al. 650 (R). MATO GROSSO: Upper Araguaia river basin, 15.VI.1966, fl., H.S. Irwin et al. 17199 (RB). Xavantina, 15.VI.1966, fl., H.S. Irwin et al. 17186 (UB).

Calyptrocarya glomerulata is recognized by its brown utricles with red trichomes adhered to white achenes. It is found in rivers. The species occurs from Central to South America.

\section{Cyperus L., Sp. Pl. 1: 44-47. 1753.}

\section{Key to species of Cyperus of the Araguaia River Basin}

1. Spikelets break up into many 1-fruit units

3.3. Cyperus odoratus

1.' Spikelets deciduous as a single fruit unit.

2. Culms not winged, spikelets spiral, glumes 7-9 nerved

3.1. C. digitatus

2'. Culms winged, spikelets fasciculate, glumes 3-5 nerved 3.2. C. haspan

3.1 Cyperus digitatus Roxb., Fl. Ind. 1: 209. 1820.

Fig. 2g-h

Plants emergent, $50-150 \mathrm{~cm}$ tall; culms not winged. Leaves 40-90×0.4-1.5 cm, glabrous, margins scabrous. Anthela $20-40 \times 20-40 \mathrm{~cm}$; internal bracts foliaceous. Spikelets $7-15 \times 0.8-1 \mathrm{~mm}$, spiral, cylindrical, deciduous as a single fruit unit; glumes $1.5-2 \times 1-1.3 \mathrm{~mm}$, oval, apex mucronate, carinate, glabrous, 7-9-nerved. Stamens 3; styles 3-fid; ovaries 3-carpellate. Achenes were not seen. Material examined: GOIÁS: rio do Peixe, 16.XI.1999, fl., C.P. Bove et al. 627 (R).

Cyperus digitatus is distinguished from other species from the Araguaia River Basin by its digitate anthelas. It was recently reported as a new occurrence for the Brazilian Cerrado (Gil et al.2007). The species was considered rare in the Araguaia River Basin due to the scarce records in herbaria (Alves et al. 2009) and to few observations in the field. It is found in swamps. The species occurs from Texas (USA) to Argentina, as well as in the Antilles and Asia.
3.2 Cyperus haspan L., Sp. Pl. 1: 45. 1753.

Fig. $2 \mathrm{i}-\mathrm{j}$

Plants emergent, $10-70 \mathrm{~cm}$ tall; culms winged. Leaves $5-20 \times 0.2-0.6 \mathrm{~cm}$ or reduced to sheaths, pilose. Anthela 1-12 $\times 0.7-14 \mathrm{~cm}$; internal bracts glumaceous. Spikelets fasciculate, $4-10 \times 1-1.5 \mathrm{~mm}$, lanceolate, deciduous as a single fruit unit; glumes $1.2-1.8 \times 0.8-1 \mathrm{~mm}$, oblong-oval, apex mucronate, glabrous, 3-5-nerved. Stamens 2-3; styles 3-fid; ovaries 3-carpellate. Achenes 0.4-0.6 $\times 0.4-0.5 \mathrm{~mm}$, trigonous, equilateral, rugulose, ovoid, white to gray. Material examined: GOIÁS: Caiapônia, 22.VI.1966, fl., D.R. Hunt 6138 (RB). Jussara, 11.XI.1999, fl. and fr., C.P. Bove et al. 484 (R). Aragarças, rio Don Bil, 11.XI.1999, fl., C.P. Bove et al. 517 (R). Britânia, 12.XI.1999, fl. and fr., C.P. Bove et al. 538 (R). MATO GROSSO: Barra do Garças, 5.V.1973, fl., W.R. Anderson 9763 (RB, UB). Jaciara, 27.IV.1981, fl., M. Sanzima \& N. Cunha s.n. (RB 342721). Nova Xavantina, 16.VII.1968, fl., G.C. Argent \& P.W. Richards 6466 (RB). TOCANTINS: Araguaína, rio das Lontras, 13.III.1968, fl., H.S. Irwin et al.21098 (UB). 

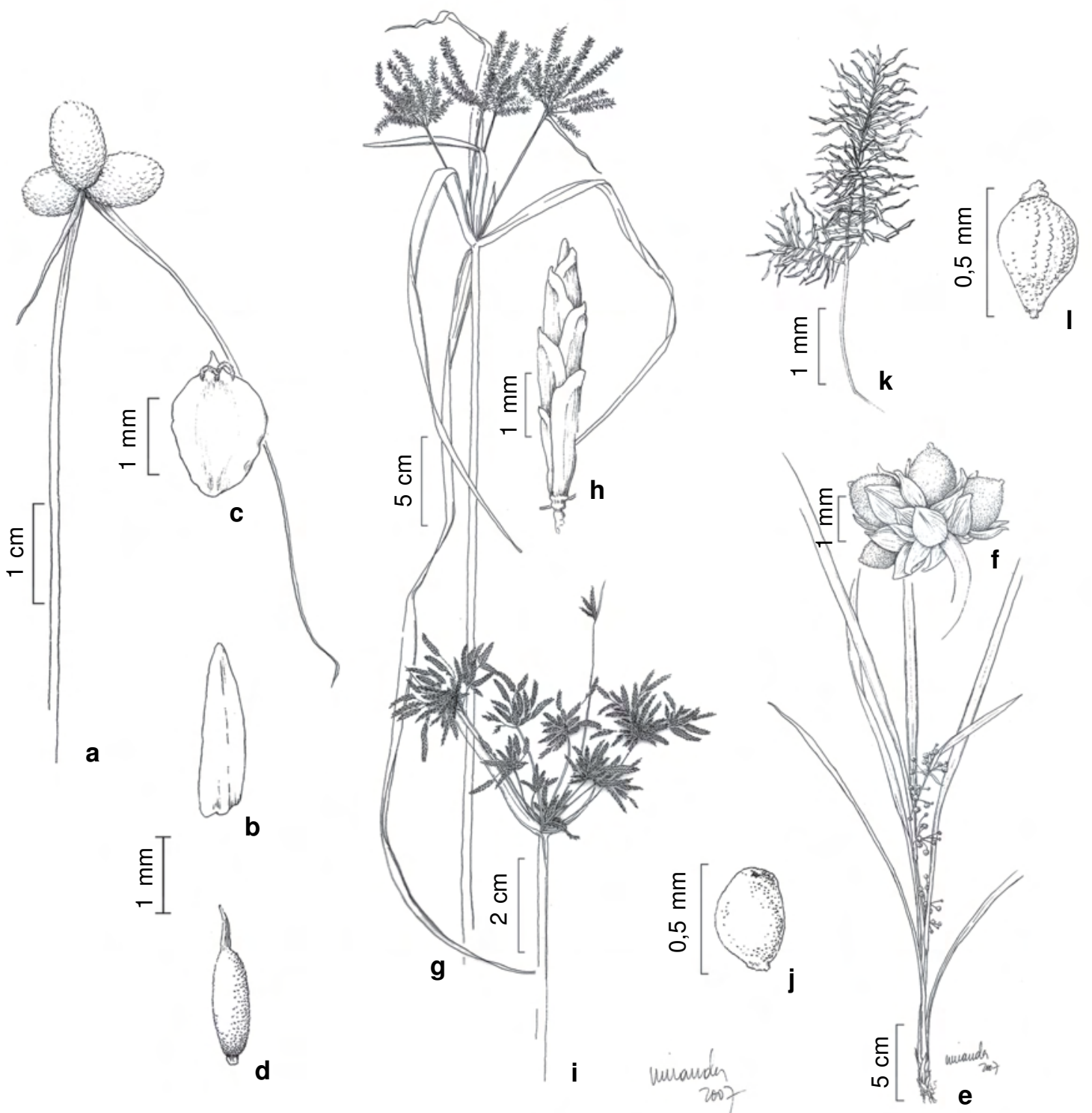

Figure 2 -a-d. Ascolepis brasiliensis (Kunth) Benth. ex C.B. Clarke - a. spikes of spikelets; b. bracteole; c. utricle (Bove et al. 1640b); d. achene (Bove et al. 548). e-f. Calyptrocarya glomerulata (Brongn.) Urb. - e. habit; f. detail of anthela (Bove et al 650). g-h. Cyperus digitatus Roxb. - g. anthelas; h. spikelet (Bove et al. 627). i-j. C. haspan L. - i. anthela; j. achene (Bove et al. 484). k-1. Cyperus odoratus L. - k. detail of anthela showing spikelets; 1. achene (Bove et al. 1659).

Cyperus haspan is easily recognized by its winged culms. It is often found in anthropic habitats. It is found in rivers, lakes and swamps with buriti palms (Mauritiaflexuosa). The species occurs from Southern United States to South America, Asia, and Africa.

\subsection{Cyperus odoratus L., Sp. Pl. 1: 46. 1753.}

Fig. 2k-1

Plants amphibious or emergent, $50-60 \mathrm{~cm}$ tall; culms not winged. Leaves $75 \times 1.5 \mathrm{~cm}$, linear- lanceolate, apex acuminate, margins and midrib scabrous. Anthela 5-29 × 18-30 cm; internal bracts foliaceous. Spikelets spiral, $7-12 \times 0.8-1 \mathrm{~mm}$, linear, deciduous as a single fruit unit; glumes 2 $3 \times 1.5-1.7 \mathrm{~mm}$, oval, apex acute, pilose, 6-nerved. Stamens 3; styles 3-fid; ovaries 3-carpellate. Achenes $1.8 \times 0.5 \mathrm{~mm}$, trigonous, equilateral, rugulose, oblong or ellipsoid, greenish brown.

Material examined: GOIÁS: Jussara, rio Água-Limpa, 11.XI.1999, fl. and fr., C.P. Bove et al. 482 (R). Aragarças, 
11.XI.1999, fl. and fr., C.P. Bove et al. 529 (R). Nova Crixás, GO-184, 14.IV.2006, fl. and fr., C.P. Bove et al. 1659 (R).

Cyperus odoratus is distinguished from other species of the Araguaia river basin by its articulated spikelets that break up into many 1 -fruit units for achene dispersal, whereas in other species the spikelets are dispersed as a single unit. It is found in rivers and swamps. The species occurs from Central United States to South America, Africa, and Madagascar.

4. Eleocharis R.Br., Prodr. 1: 224. 1810.

\section{Key to species of Eleocharis of the Araguaia River Basin}

1. Culms septate

4.5. Eleocharis interstincta

1'. Culms not septate.

2. Spikes uniflorous 4.3. E. capillacea

2'. Spikes multiflorous.

3. Glumes not carinate.

4. Culms trigonous; apex of sheath obtuse 4.1. E. acutangula

4. Culms cylindrical, 4- or 5-angled; apex of sheath acuminate.

5. Glumes cartilaginous, achenes cancellate, tubercles conical.... 4.8. E. plicarhachis

5'. Glumes membranous, achenes smooth, tubercles pyramidal

4.7. E. nudipes

3'. Glumes carinate.

6. Sheaths with hyaline appendix, apex mucronate

4.10. E. sellowiana

6'. Sheaths without hyaline appendix, apex acuminate, acute or obtuse.

7. Glumes cartilaginous, tubercles pyramidal.

7'. Glumes membranous, tubercles conical

4.6. E. nana

8. Achenes cancellate, with bristles 4.9. E. retroflexa

8'. Achenes smooth.

9. Achenes without bristles

4.2. E. bicolor

9'. Achenes with bristles 4.4. E. filiculmis

4.1 Eleocharis acutangula (Roxb.) Schult., Mant. 2:91. 1824. Figs. 3a-c, $7 \mathrm{a}$

Plants emergent, $17-90 \mathrm{~cm}$ tall; culms trigonous, 2-6 mm wide, not septate. Sheaths 6.5$22 \mathrm{~cm}$ long, apex obtuse. Spikes multiflorous, 15$35 \times 2.5-5 \mathrm{~mm}$, lanceolate to narrowly oblong; glumes spiral, $3-4.5 \times 2-3 \mathrm{~mm}$, oval, apex acute to obtuse, not carinate, cartilaginous, margins hyaline; fertile glumes numerous; sterile glumes absent; . Perianth with 6-7 bristles, 1-2.5 mm long, retrorsely scabrous; stamens 3; styles 3-fid; ovaries 3carpellate. Achenes 1.3-1.7 × 1.2-1.5 mm, obovoid to orbicular, biconvex, greenish brown to light brown, reticulate; tubercles $0.4-0.6 \times 0.7-$ $1 \mathrm{~mm}$, pyramidal.

Material examined: GOIÁS: Aruanã, 14.X.1997, fl. and fr., C.P. Bove et al. 290 (R). Aragarças, 26.V.1997, fl. and fr., C.P. Bove et al. 186 (R). Aruanã, 15.XI.1999, C.P. Bove et al. 601 (R).

Eleocharis acutangula is easily distinguished by its trigonous culms. It is found in buriti-palm swamps (Mauritia flexuosa), locally known as buritizais, and in temporary ponds. The species occurs from
Mexico to South America, Asia, Africa, Madagascar, and Oceania.

4.2 Eleocharis bicolor Chapm., Fl. South. U.S. 517. 1860.

Figs. 3d-e, $7 b$

Plants emergent, $3-12 \mathrm{~cm}$ tall; culms cylindrical to 4-angled, $0.2-0.5 \mathrm{~mm}$ wide, not septate. Sheaths $0.5-1.8 \mathrm{~cm}$ long, membranous, apex acute, acuminate or obtuse. Spikes multiflorous, 3-4 × 1-1.5 mm, lanceolate to ellipsoid, sometimes obovate; glumes spiral or distichous, 1 sterile, numerous fertile, 1-1.6 $\times 0.8-1 \mathrm{~mm}$, widely oval, apex obtuse, emarginate, carinate, cartilaginous, margins membranous. Perianth absent, sometimes with vestigial bristles; stamens 2; styles 2-3 fid; ovaries 2-3 carpellate. Achenes $0.6-0.8 \times 0.4-0.6 \mathrm{~mm}$, obovoid, biconvex or trigonous, light green to white, shiny, reticulate or smooth; tubercles $0.1-0.2 \times 0.2-0.3 \mathrm{~mm}$, pyramidal. Material examined: GOIÁS Aruanã, 15.XI.1999, fl. and fr., C.P. Bove et al. 592 (R).

Eleocharis bicolor is often mistaken for E. nana. The former has rigid culms, achenes without bristles and pyramidal tubercles, whereas E. nana has flaccid 


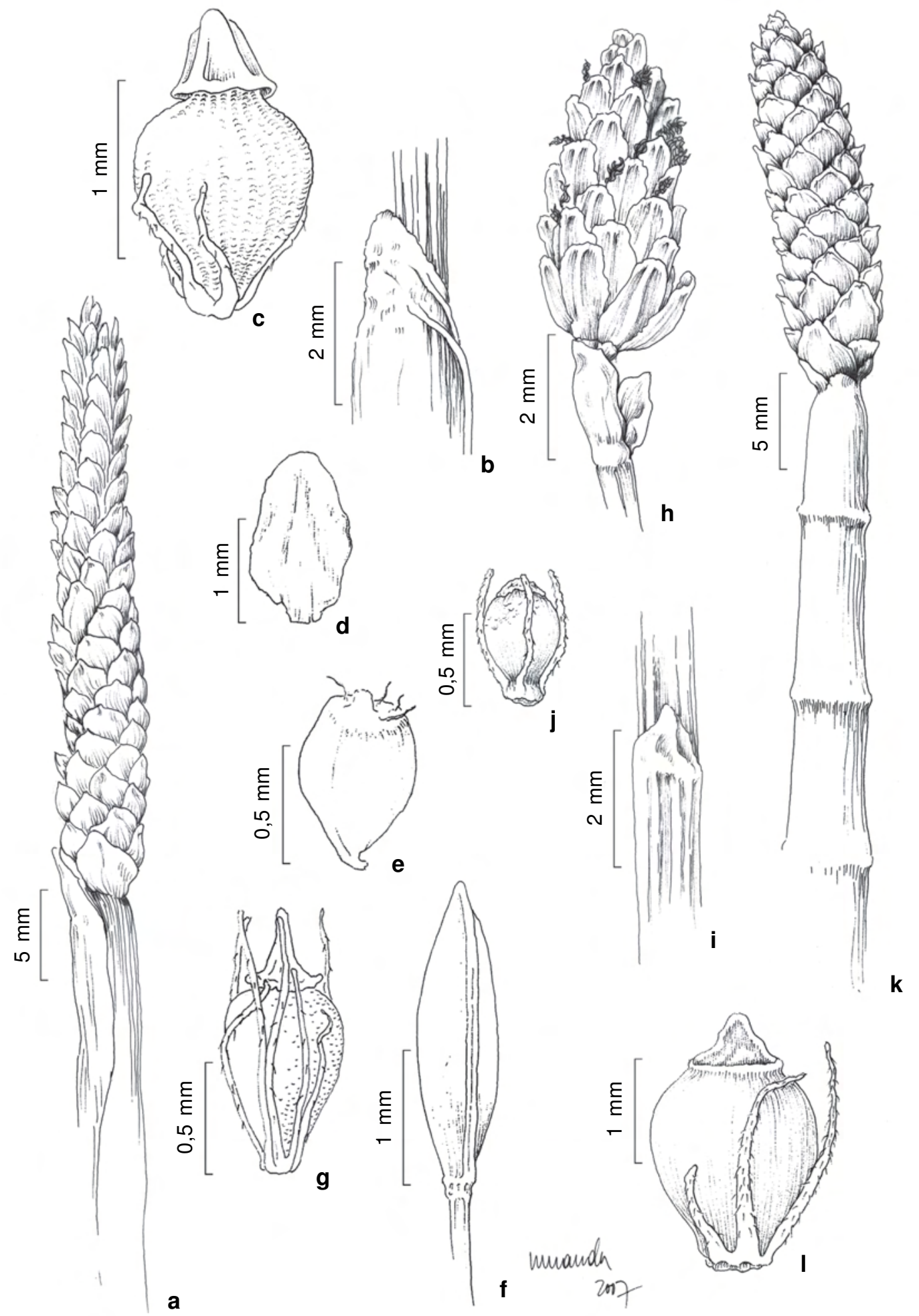

Figure 3 - a-c. Eleocharis acutangula (Roxb.) Schult. - a. spike and culm not septate; b. apex sheath; c. achene (Bove et al. 186). d-e. E. bicolor Chapm. - d. fertile glume; e. achene (Bove et al. 592). f-g. E. capillacea Kunth -f. spike; g. achene (Bove et al. 199b). h-j. E. filiculmis Kunth - h. spike; i. apex sheath; j. achene (Bove et al. 184). k-1. E. interstincta (Vahl) Roem. \& Schult. - k. spike and septate culm; 1. achene (Bove et al. 552). 
culms, achenes with bristles and conical tubercles. E. bicolor is found in temporary ponds. The species occurs from southern USA to South America.

4.3 Eleocharis capillacea Kunth, Enum. Pl. 2: 139. 1837.

Figs. 3f-g, 7c

Plants emergent or fixed submerged, $2-5 \mathrm{~cm}$ tall; culms 4-angled, $0.2-0.3 \mathrm{~mm}$ wide, not septate. Sheaths $0.4-0.5 \mathrm{~cm}$ long, membranous, apex obtuse. Spikes 1$3 \times 0.5-0.8 \mathrm{~mm}$, uniflorous, lanceolate to narrowly ellipsoid; glumes distichous, 1 sterile, 1 fertile, $2-3 \times$ $0.5-0.6 \mathrm{~mm}$, narrowly oblong, apex obtuse, carinate, membranous. Perianth with 5 bristles, $0.5-0.8 \mathrm{~mm}$ long, retrorsely scabrous; stamens 2 ; styles 2-fid; ovaries 2-carpellate. Achenes $0.8-1 \times 0.6-0.8 \mathrm{~mm}$, obovoid, biconvex, brown or black, smooth; tubercles $0.6-0.8$ $\times 0.2-0.4 \mathrm{~mm}$, conical.

Material examined: GOIÁS: Jussara, 28.V.1997, fl. and fr., C.P. Bove et al.199b (R). TOCANTINS: Araguaína, $7^{\circ} 12^{\prime}$ S 48 $14^{\circ}$ 'W, fl., G. Eiten \& L.T. Eiten 10165 (UB). Additional material examined: GOIÁS: Itarumã, GO-202, 15.I.2007, fl. and fr., C.P. Bove et al. 1819 (R). MATO GROSSO: Paranatinga, 9.II.1993, fl. and fr., C.P. Bove et al. 88 (R). MATO GROSSO DO SUL: Sonora, 22.VII.2003, fl. and fr., C.P. Bove et al.1153 (R). RONDÔNIA: P. Bueno, 07.XI.1979, fl. and fr., M.G. Vieira et al. 983 (RB).

Eleocharis capillacea is a small plant that can be recognized by its thin hairy appearance. It is found in rivers and swamps. The species occurs from Central to South America.

4.4 Eleocharis filiculmis Kunth, Enum. Pl. 2: 144. 1837.

Figs. 3h-j, $7 \mathrm{~d}$

Plants emergent, $15-50 \mathrm{~cm}$ tall; culms 5-angled, $0.8-1.2 \mathrm{~mm}$ wide, not septate. Sheaths $2-3 \mathrm{~cm}$ long, cartilaginous, apex acuminate. Spikes multiflorous, $5-10 \times 1.2-3 \mathrm{~mm}$, narrowly obovoid to lanceolate; glumes spiral, 1 sterile, numerous fertile, $1.5-2 \times 1-$ $1.3 \mathrm{~mm}$, oval, apex obtuse, carinate, cartilaginous, margins hyaline. Perianth with 5-6 bristles, $0.8-0.9 \mathrm{~mm}$ long, retrorsely scabrous; stamens 3 ; styles 3-fid, ovaries 3 -carpellate. Achenes $0.8-1 \times 0.5-0.6 \mathrm{~mm}$, obovoid, trigonous, light green to yellowish, smooth; tubercles $0.3-0.4 \times 0.3-0.4 \mathrm{~mm}$, pyramidal.

Material examined: Goiás: Aragarças, 26.V.1997, fl. and fr., C.P. Bove et al. 184 (R). Jussara, 12.XI.1999, fl. and fr., C.P. Bove et al. 544 (R). TOCANTINS: Araguaína, $7^{\circ} 12^{\prime}$ 'S 48 $14^{\prime}$ 'W, fl., G. Eiten \& L.T. Eiten 10156 (UB).

Eleocharis filiculmis is distinguished by its pentangular culm. See comments for E. sellowiana. E. filiculmis is found in lakes and buriti-palm swamps (Mauritia flexuosa L.f.), locally known as buritizais. The species occurs from Mexico to South America.
4.5 Eleocharis interstincta (Vahl) Roem. \& Schult., Syst. Veg. 2: 149. 1817.

Figs. 3k-1, 7e

Plants emergent, $26-80 \mathrm{~cm}$ tall; culms cylindrical; 5-8 mm wide, septate. Sheaths 13$25 \mathrm{~cm}$ long, membranous, apex acuminate. Spikes multiflorous, $14-38 \times 5-7 \mathrm{~mm}$, lanceolate to narrowly ellipsoid, sometimes oblong; glumes spirally arranged, 1 sterile, numerous fertile, $5-7 \times 2.5-4 \mathrm{~mm}$, oval, apex obtuse, not carinate, cartilaginous, margins hyaline. Perianth with $7-8$ bristles, $2-3 \mathrm{~mm}$ long, retrorsely scabrous; stamens 3 ; styles $2-3$ fid; ovaries 2-carpellate. Achenes 1.8-2 $\times 1.5-1.8 \mathrm{~mm}$, obovoid to orbicular, biconvex, green to light green, sometimes yellowish, costate, reticulate; tubercles $0.6-1 \times 0.8-1 \mathrm{~mm}$, pyramidal.

Material examined: GOIÁS: Aruanã, 14.X.1997, fl. and fr., C.P. Bove et al. 291 (R). Jussara-Britânia road (via Jacilândia) $58.9 \mathrm{~km}$ from Jussara, 12.XI.1999, fl. and fr., C.P. Bove et al. 552 (R). MATO GROSSO: Nova Xavantina, $14^{\circ} 38^{\prime}$ 'S 52 ${ }^{\circ} 14^{\prime} \mathrm{W}, 16 . \mathrm{I} .1968$, fl. and fr., D. Philcox \& A. Ferreira 4089 (RB).

Eleocharis interstincta is distinguished from other species of the Araguaia River Basin by its septate culms. It is found in rivers and temporary ponds. The species occurs from the USA to South America.

4.6. Eleocharis nana Kunth, Enum. Pl. 2: 140.1837.

Figs. 4a-d, $7 f$

Plants emergent or fixed-submerged, 3-7 cm tall; culms 4-angled, $0.2-0.3 \mathrm{~mm}$ wide, not septate. Sheaths $0.5-2 \mathrm{~cm}$ long, membranous, apex obtuse. Spikes multiflorous, 4-5 × 1-1.5 mm, lanceolate; glumes spiral, 2 sterile, numerous fertile, $1-2.5 \times 0.5$ $0.8 \mathrm{~mm}$, oval, apex obtuse, carinate, membranous, midrib thickened. Perianth with 5 bristles, $1-1.5 \mathrm{~mm}$ long, retrorsely scabrous; stamens 3; styles 3-fid; ovaries 3-carpellate. Achenes $0.4-0.7 \times 0.7-1 \mathrm{~mm}$, trigonous, obovoid, green, smooth; tubercles 0.1$0.2 \times 0.2-0.3 \mathrm{~mm}$, conical.

Material examined: MATO GROSSO: Santa Cruz, 1891/1892, fl. and fr., S. Moore 530 (R).

Additional material examined: BAHIA: Ilhéus, 21.IV.1981, fl. and fr., S.A. Mori et al. s.n. (RB 246955). RIO DE JANEIRO: Restinga de Jacarepaguá, 23.VI.1969, fl. and fr., D. Sucre et al. 5377 (RB). SÃO PAULO: Itanhaém, 6.XI.2000, fl. and fr., C.P. Bove et al. 759 (R).

Eleocharis nana was recently reported as a new occurrence for the Brazilian Cerrado (Gil et al. 2007). The species was considered rare in the Araguaia River Basin due to scarce records in herbaria and few observations in the field. See comments for E. bicolor. E. nana occurs from the USA to Uruguay. 

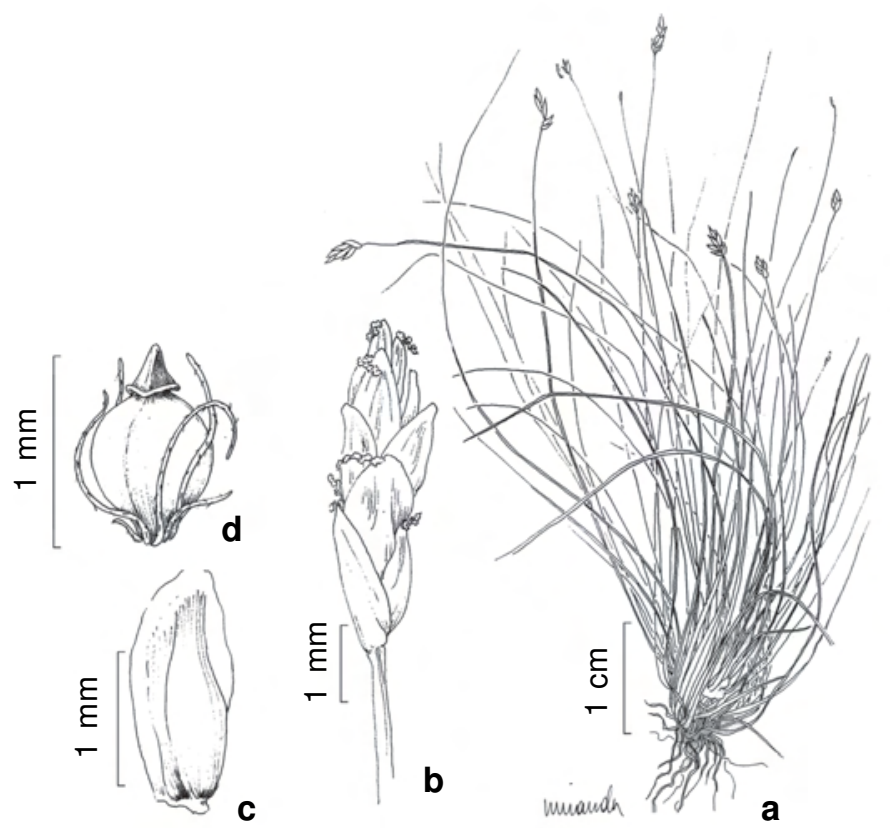
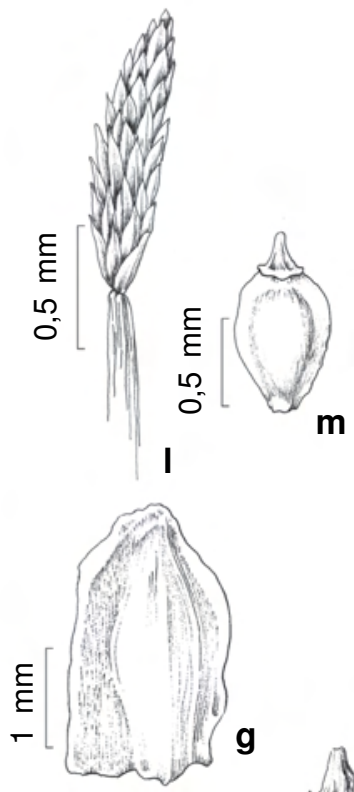
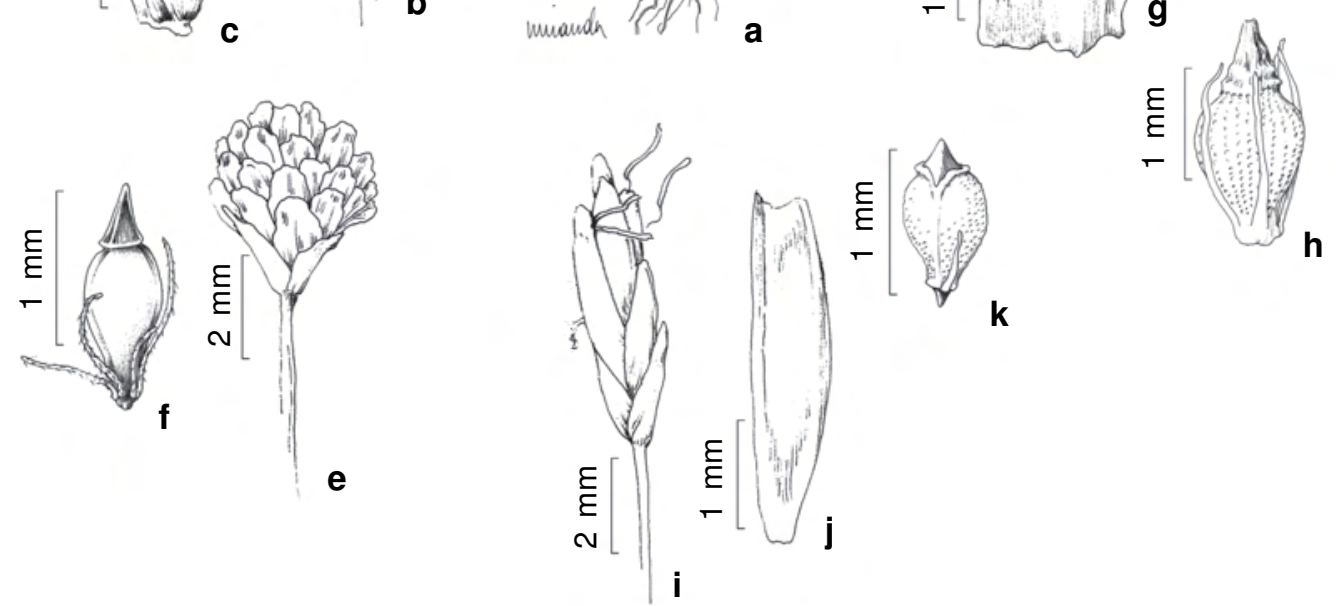

Figure 4 - a-d. Eleocharis nana Kunth - a. habit; b. spike; c. fertile glume; d. achene (Moore 530). e-f. E. nudipes (Kunth) Palla-e. spike; f. achene (Bove et al. 568). g-h. E. plicarhachis (Griseb.) Schult. - g. fertile glume; h. achene (Bove et al. 230b). i-k. E. retroflexa (Poir.) Urb. - i. spike; j. fertile glume; k. achene (Bove et al. 1649). 1-m. E. sellowiana Kunth - 1. spike; m. achene (Bove et al. 1326).

4.7 Eleocharis nudipes (Kunth) Palla, Denkschr. Kaiserl. Akad. Wiss., Wien. Math.-Naturwiss. Kl. 79: 171.1908. Figs. 4e-f, $7 \mathrm{~g}$

Plants emergent, $15-90 \mathrm{~cm}$ tall; culms cylindrical, 1-2.5 mm wide, not septate. Sheaths 4$12 \mathrm{~cm}$ long, cartilaginous, apex acuminate, apiculate. Spikes multiflorous, $3-7 \times 2-5 \mathrm{~mm}$, ellipsoid to obovoid; glumes spiral, 2 sterile, numerous fertile, $2.3-3.3 \times 1-1.2 \mathrm{~mm}$, oblong, apex obtuse, not carinate, membranous. Perianth with 4-6 bristles, $0.5-1.5 \mathrm{~mm}$ long, retrorsely scabrous; stamens 3; styles 2-3 fid; ovaries 2-3 carpellate.
Achenes 1-1.3 $\times 0.7-0.8 \mathrm{~mm}$, obovoid to orbicular, biconvex or trigonous, light green to light brown, smooth or weakly reticulate; tubercles $0.3-0.6 \times$ $0.5-0.7 \mathrm{~mm}$, pyramidal.

Material examined: GOIÁS: Aragarças, 26.V.1997, fl. and fr., C.P. Bove et al. 185 (R); Jussara, 12.XI.1999, fl. and fr., C.P. Bove et al. $568(\mathrm{R})$.

Eleocharis nudipes is easily recognized by its obovoid inflorescence and acuminate sheath with apiculus. It is found in buriti palm swamps (Mauritia flexuosa). The species occurs from central Brazil to Uruguay. 
4.8 Eleocharis plicarhachis (Griseb.) Svenson, Rhodora 31:158.1929. Figs. 4g-h, 7h

Plants emergent, $23-52 \mathrm{~cm}$ tall; culms cylindrical, 1-5 mm wide, not septate. Sheaths 5.1$8.5 \mathrm{~cm}$ long, membranous, apex acuminate. Spikes multiflorous, $10-27 \times 2-4 \mathrm{~mm}$, lanceolate; glumes spiral, none sterile, numerous fertile, 3-3.5 $\times 1.5-$ $2 \mathrm{~mm}$, oval, apex obtuse, not carinate, cartilaginous, margins hyaline. Perianth with 6-7 bristles, 1.5-2 $\mathrm{mm}$ long, retrorsely scabrous; stamens 1-2; styles 2-fid; ovaries 2-carpellate. Achenes 1-1.2 $\times 0.8-1 \mathrm{~mm}$, obovoid to orbicular, biconvex, greenish brown to yellowish, cancellate; tubercles $0.5-0.7 \times 0.5 \mathrm{~mm}$, conical.

Material examined: GOIÁS: Aragarças, 9.X.1997, fr., C.P. Bove et al. $230 b$ (R).

Additional material examined: DISTRITO FEDERAL: Reserva Ecológica IBGE, 14.XI.1985, fl. and fr., R.C. Mendonça 581 (RB).

Eleocharis plicarhachis may be mistaken for E. nudipes. The former has cartilaginous glumes and cancellate achenes, whereas E. nudipes has membranous glumes and smooth achenes. E. plicarhachis is found in buriti palm swamps (Mauritia flexuosa), locally known as buritizais, and occurs from Mexico to Paraguay.

4.9 Eleocharis retroflexa (Poir.) Urb., Symb. Antill. 2: 165.1900.

Figs. 4i-k, $7 \mathrm{i}$

Plants emergent, $5-20 \mathrm{~cm}$ tall; culms cylindrical, $0.2-0.4 \mathrm{~mm}$ wide, not septate. Sheaths $1-2 \mathrm{~cm}$ long, cartilaginous, apex membranous, acute or obtuse. Spikes multiflorous, $5-7 \times 1.5-3 \mathrm{~mm}$, lanceolate to ellipsoid; glumes distichous, 1 sterile, numerous fertile, $3-4 \times 1-1.5 \mathrm{~mm}$, narrowly oblong, apex emarginate, carinate, cartilaginous, margins hyaline. Perianth with 6 bristles, $0.3-0.6 \mathrm{~mm}$ long, antrorsely scabrous at the apex; stamens 3; styles 3-fid; ovaries 3-carpellate. Achenes $0.7-1 \times 0.6-0.8 \mathrm{~mm}$, obovoid, trigonous, green, costate, cancellate; tubercles $0.1-$ $0.2 \times 0.5-0.7 \mathrm{~mm}$, pyramidal.

Material examined: GOIÁS: Jussara, 28.V.1997, fl. and fr., C.P. Bove et al. 199a (R). Aruanã, 13.IV.2006, fl. and fr., C.P. Bove et al. $1649(\mathrm{R})$.

Additional material examined: RIO DE JANEIRO: Jacarepaguá, 16.VI.1969, fl. and fr., D. Sucre 5347 (RB).

Eleocharis retroflexa is easily recognized by its red glumes and white filaments. It is found in temporary ponds. The species occurs from Central to South America, as well as in Africa and Madagascar.

4.10 Eleocharis sellowiana Kunth, Enum. Pl. 2: 149. 1837. Figs. 41-m, 8a

Plants emergent, $20-50 \mathrm{~cm}$ tall; culms cylindrical, 0.5-2 mm wide, not septate. Sheaths 5$12 \mathrm{~cm}$ long, cartilaginous, apex mucronate with hyaline appendix. Spikes multiflorous, 5-10 × 2-4 mm, lanceolate; glumes spiral, 2 sterile, numerous fertile, 2-3 $\times 0.8-1 \mathrm{~mm}$, oblong to lanceolate, apex obtuse, carinate, membranous. Perianth with $7-8$ bristles, $0.5-$ $1 \mathrm{~mm}$ long or absent, retrorsely scabrous; stamens 2 ; styles 2-fid; ovaries 2-carpellate. Achenes $0.8-1 \times$ 0.6-0.8 mm, obovoid, biconvex, olivaceous, smooth; tubercles $0.3-0.4 \times 0.3-0.4 \mathrm{~mm}$, conical.

Material examined: GOIÁS: Caiapônia, 27.VI.1966, fl. and fr., D.R. Hunt 6242 (RB). MATO GROSSO: Alto Araguaia, 14.I.2004, fl. and fr., C.P. Bove et al. 1326 (R).

Eleocharis sellowiana can be mistaken for E. filiculmis. The former has sheaths with mucronate apex and hyaline appendix, whereas $E$. filiculmis has sheaths with acuminate apex without appendix. E. sellowiana is found in buriti palm swamps (Mauritia flexuosa), and occurs from Central to South America.

5. Fimbristylis Vahl, Enum. Pl. 2: 285. 1805.

\section{Key to species of Fimbristylis of the Araguaia River Basin}

1. Leaves distichous 5.3. Fimbristylis miliacea

1'. Leaves spiral.

2. Spikelets $0.7-1 \mathrm{~mm}$ wide, achenes cordate, not stipitate 5.1. F. aestivalis

2'. Spikelets 10-20 mm wide, achenes obovoid, stipitate 5.2. F. dichotoma

5.1 Fimbristylis aestivalis (Retz.) Vahl, Enum. Pl. 2: 288. 1806.

Figs. 5a-b, 8b

Plants emergent, 7-25 cm tall; culms trigonous, $0.5-0.7 \mathrm{~mm}$ wide. Leaves 3-ranked, 30$140 \times 0.5-1 \mathrm{~mm}$, linear, apex acute, margins apically scabrous, not ligulate, rarely pilose. Anthela 3-5 $\times 2-4 \mathrm{~cm}$. Spikelets $2.5-6 \times 0.7-1 \mathrm{~mm}$, ovallanceolate to oblong; glumes $1-1.5 \times 0.5 \mathrm{~mm}$, oval, apex mucronate, reflexed. Stamens 1; styles 2-fid, not fimbriate; ovaries 2-carpellate. Achenes $0.5 \times$ 0.4-0.5 mm, biconvex, cordate, light green, shiny, reticulate, not stipitate. 
Material examined: GOIÁS: Mozarlândia, 15.XI.1999, fl. and fr., C.P. Bove et al. 608 a (R). MATO GROSSO: rio das Mortes, 16.XI.1968, fl. and fr., R.M. Harley \& $R$. Souza 11105 (RB).

Fimbristylis aestivalis in vegetative state can be mistaken for $F$. dichotoma. The former has spread leaves and many anthelas, whereas $F$. dichotoma has more compact leaves and few anthelas. $F$. aestivalis is found in rivers and swamps, and occurs from Central to South America.

5.2 Fimbristylis dichotoma (L.) Vahl, Enum. Pl. 2: 287. 1805.

Figs. $5 c-d, 8 c$

Plants emergent, $30-70 \mathrm{~cm}$ tall; culms cylindrical, 0.8-1.5 mm wide. Leaves 3-ranked, 100$350 \times 1-3.5 \mathrm{~mm}$, linear, apex acute, margins scabrous. Ligule absent or present, pilose. Anthela $2-5 \times 1.5-$ $4 \mathrm{~cm}$. Spikelets $5-12 \times 10-20 \mathrm{~mm}$, lanceolate; glumes $2.5-3 \times 1.2-1.5 \mathrm{~mm}$, oblong to oval, apex acute. Stamens 1; styles 2-fid, fimbriate; ovaries 2carpellate. Achenes $0.9-1 \times 0.7-0.9 \mathrm{~mm}$, biconvex, obovoid, white to beige, shiny, reticulate, stipitate. Material examined: Goiás: Jussara, 11.XI.1999, fl. and fr., C.P. Bove et al. 486 (R). Aragarças, 11.XI.1999, fl. and fr., C.P. Bove et al. $510(\mathrm{R})$.

See comments for Fimbristylis aestivalis. F. dichotoma is found in rivers and swamps, and occurs from the USA to South America, as well as in Africa, Madagascar, Asia, and Australia.

5.3 Fimbristylis miliacea (L.) Vahl, Enum. Pl. 2:287. 1805.

Figs. 5e, $8 \mathrm{~d}$

Plants emergent, 15-73 cm tall; culms 4angled, 1-2 mm wide. Leaves distichous, 100-400 $\times 2-3 \mathrm{~mm}$, linear, apex obtuse, trichomes sparse, margins and midrib scabrous. Ligule absent. Anthela $2-10 \times 1-5 \mathrm{~cm}$. Spikelets $2-3 \times 1-2 \mathrm{~mm}$, ovoid; glumes $1-1.5 \times 0.2-0.3 \mathrm{~mm}$, oval, apex obtuse. Stamens 2; styles 2-3 fid, fimbriate; ovaries 2-3 carpellate. Achenes $0.5-1 \times 0.3-0.4 \mathrm{~mm}$, biconvex or trigonous, obovoid, green, reticulateverrucose, shiny, not stipitate.

Material examined: GOIÁS: Caiapônia, Serra do Caiapó, 30.IV.1973, fl. and fr., W.R. Anderson 9521 (R, UB). MATO GROSSO: Rio das Mortes, 16.XI.1968, fl. and fr., R.M. Harley \& R. Souza 11111 (RB). TOCANTINS: Araguaína, rio das Lontras, 13.III.1968, fl., H.S. Irwin et al. 21109 (RB, UB). Formoso do Araguaia, BR-242, 15.IV.2006, fl. and fr., C.P. Bove et al. 1668 (R).

Fimbristylis miliacea is often found in anthropic habitats. It is found in rivers and swamps. The species occurs from southern USA to South America, as well as in Africa, Asia, and Europe.
6. Fuirena Rottb., Descr. Icon Rar. Pl.70. 1773. 6.1 Fuirena umbellata Rottb., Descr. Icon. Rar. Pl. 70. pl 19, Fig. 3. 1773.

Figs. 5f-h

Plants emergent, 65-95 cm tall. Leaves (6-)9$18 \times 0.6-1.5 \mathrm{~cm}$, linear-lanceolate, apex acute, pilose or glabrous; ligules membranous, scarious. Anthela 7-8 lateral, $0.6-2.5 \times 1-4 \mathrm{~cm}$. Petals obovate, apex aristate, 3-nerved, membranous. Achenes $0.7-1.2 \times 0.5-0.8 \mathrm{~mm}$, obovoid, white, light green or yellowish.

Material examined: GOIÁS: Aragarças, 11.XI.1999, fl. and fr., C.P. Bove et al. 522 (R). Britânia, 12.XI.1999, fl. and fr., C.P. Bove et al. 540 (R). Aragarças, Córrego do Gato, $15^{\circ} 51^{\prime}$ 'S $52^{\circ} 15^{\prime} \mathrm{W}, 17 . X .1968$, fl. and fr., R.M. Harley et al. 10716 (UB). Mozarlândia, 19.I.2007, fl. and fr., C.P. Bove et al. 1831 (R). MATO GROSSO: Barra do Garças, 5.V.1973, W.R. Anderson 9772 (UB).

Fuirena umbellata is easily recognized by its ligulate leaves along the stem, its reddish brown glumes with green nerves and its petaloid perianth. It is found in rivers, lakes and buriti palm swamps (Mauritia flexuosa), and in temporary ponds. The species occurs from Central to South America, as well as in Africa and Madagascar.

7. Lipocarpha R. Br., Narr. Exped. Zaire 5: 459. 1818. 7.1 Lipocarpha humboldtiana Nees, Linnaea 9: 287. 1834.

Fig. 5i-k

Plants emergent, $15-50 \mathrm{~cm}$ tall. Leaves 3-16 $\times 0.1 \mathrm{~cm}$, linear, apex obtuse, margins scabrous at the apex. Spikes multiflorous, 3-10 × 2-7 mm; external bracts $2-30 \times 0.05-1 \mathrm{~mm}$; bracteoles $3-8 \times$ $3 \mathrm{~mm}$, oval-triangular, apex acuminate, glabrous; glumes $1.8 \times 1.6 \mathrm{~mm}$, elliptic, apex acute. Achenes 1-1.5 $\times 0.4-0.5 \mathrm{~mm}$, oblong, oblong-ovoid, brown or green, rugulose.

Material examined: GOIÁS: Palestina, GO-221, 11.IV.2006, fl. and fr., C.P. Bove et al. 1640 (R). Santa Rita do Araguaia, 30.III.2000, fl. and fr., D.M.S. Rocha 253 (UB).

Additional material examined: MATO GROSSO: Cárceres, 22.X.1984, fl. and fr., M. Emmerich et al. 5190 (R). PARANÁ: Jaguariava, 17.I.1965, fl. and fr., L.B. Smith et al. 14697 (R). RIO GRANDE DO SUL: 4.IV.1902, fl., G.A. Malme $622 a$ (R). SANTA CATARINA: Chapecó, 26.XII.1956, L.B. Smith et al. 9480 (R). Lajes, 1.I.1957, fl. and fr., L.B. Smith \& R. Reitz 10064 (R). SÃO PAULO: fl. and fr., M. Gaudichaud 50 (R).

See comments for Ascolepis brasiliensis. L. humboldtiana is found in buriti palm swamps (Mauritia flexuosa L.f.), locally known as buritizais. The species occurs from Central to South America. 


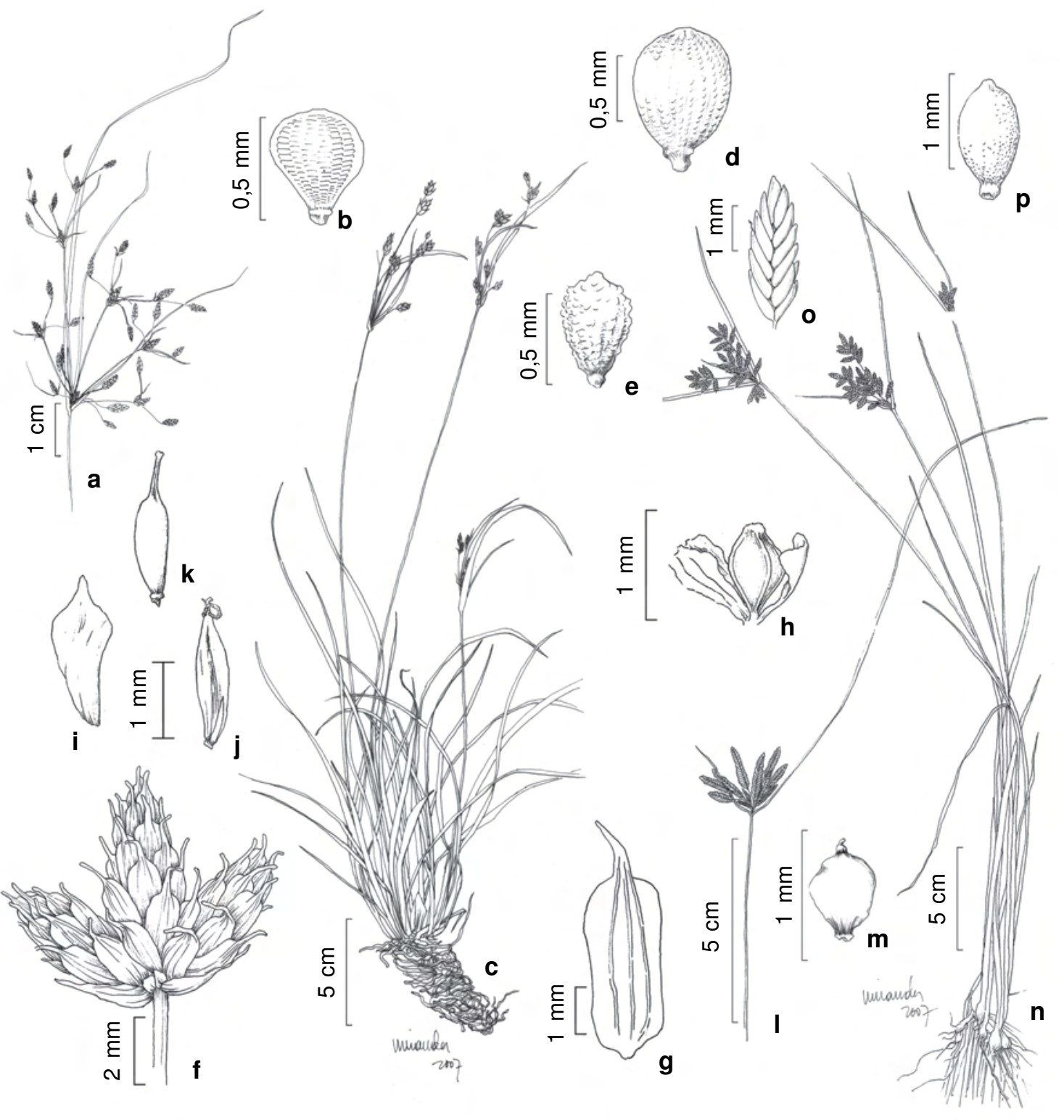

Figure 5 - a-b. Fimbristylis aestivalis (Retz.) Vahl - a. detail of anthela; b. achene (Bove et al. 608a). c-d. F. dichotoma (L.) Vahl - c. habit; d. achene (Bove et al. 486). e. F. miliacea (L.) Vahl - e. achene (Bove et al. 1668). fh. Fuirena umbellata Rottb. - f. detail of anthela; g. glume; h. achene and perianth (Bove et al. 540). i-k. Lipocarpha humboldtiana Nees - i. bracteole; j. flower enwrapped by glume and prophyll; $\mathrm{k}$. achene (Bove et al. 1640). 1-m. Pycreus lanceolatus (Poir.) C.B. Clarke - 1. detail of anthela showing spikelets; m. achene (Bove et al. 1830). n-p. Pycreus unioloides R.Br. - n. habit; o. spikelet; p. achene (Bove et al. 518 R). 
8. Pycreus P. Beauv., Fl. Oware 2: 48. 1816.

\section{Key to species of Pycreus of the Araguaia River Basin}

1. Spikelets fasciculate; achenes smooth, inequilateral

8.1. Pycreus lanceolatus

1'. Spikelets spirally arranged; achenes papillose, equilateral 8.2. P. unioloides

8.1 Pycreus lanceolatus (Poir.) C.B. Clarke in Durand \& Schinz, Consp. Fl. Afr. 5:538. $1894 . \quad$ Fig. 5l-m

Plants amphibious or emergent, $39-57 \mathrm{~cm}$ tall; culms not winged. Leaves $13-27 \times 0.1 \mathrm{~cm}$, linear, apex acute, margins scabrous. Anthela $0.8-4 \times$ $0.5-4.5 \mathrm{~cm}$; internal bracts absent. Spikelets fasciculate, $3-9 \times 3 \mathrm{~mm}$, lanceolate, deciduous as a single fruit unit; glumes $2-2.2 \times 1-1.5 \mathrm{~mm}$, oval, apex acute, mucronate, glabrous, 1 -nerved. Stamens 2; styles 2-fid; ovaries 2-carpellate. Achenes $0.8-1 \times 0.3-0.8 \mathrm{~mm}$, biconvex, inequilateral, smooth, obovoid, brown or black.

Material examined: GOIÁS: Mozarlândia, $14^{\circ} 28^{\prime} 27.5^{\prime}$ 'S $50^{\circ} 28^{\prime} 21.7^{\prime \prime} \mathrm{W}, 19 . \mathrm{I} .2007$, fl. and fr., C.P. Bove et al. $1830(\mathrm{R})$.

Additional material examined: MINAS GERAIS: Carandaí, 15.II.1962, fl. and fr., A.P. Duarte 6342 (RB). Northern Minas Gerais, 22.XI.1962, fl. and fr., A.P. Duarte 7433 (RB). Serra do Anta, 7.II.1970, fl. and fr., H.S. Irwin et al 26280 (RB). Serra do Cabral, 9.II.1970, fl. and fr., H.S Irwin et al. 27182 (RB). Serra do Espinhaço, 17.II.1969, fl. and fr., H.S Irwin et al. 23434 (RB). Serra de São José (São José Mountain Range), 6.III.1998, fl. and fr., R.J. Valka-Alves 230 (RB).

The species was considered rare in the Araguaia River Basin due to scarce records in herbaria and few observations in the field. It is distinguished from other species of the Araguaia River Basin by its inequilateral achenes. It is found in temporary ponds. The species occurs from Southern United States to South America, Africa, and Madagascar.

8.2 Pycreus unioloides R. Br., Prodr. 216. 1810.

Fig. 5n-p

Plants amphibious or emergent, $36-62 \mathrm{~cm}$ tall; culms not winged. Leaves $16-36 \times 0.2-0.4 \mathrm{~cm}$, linearlanceolate, glabrous. Anthela 1-10 × 1.5-17 cm; internal bracts absent. Spikelets $1-2 \times 0.3-0.6 \mathrm{~cm}$, spiral, elliptic, deciduous as a single fruit unit; glumes 3.5-4 × 1-2 mm, oval, apex apiculate, glabrous, 3-nerved. Stamens 3; styles 2-fid; ovaries 2-carpellate. Achenes 1-1.5 ×0.8-1 mm, biconvex, equilateral, papillose, obovoid, greenish brown to gray.

Material examined: GOIÁS: Aragarças, 11.XI.1999, fl. and fr., C.P. Bove et al. 518 (R).

Additional material examined: GOIÁS: Alto Paraíso, Chapada dos Veadeiros, 19.III.1973, fl. and fr., W.R. Anderson 7456 (RB). PARANÁ: Lapa, 3.III.1970, fl. and fr., T. Koyama et al. s.n. (RB 305605).

Pycreus unioloides is distinguished from other Pycreus species of the Araguaia River Basin by its reddish brown sheaths of leaves. It is found in buriti palm swamps (Mauritia flexuosa). The species occurs from Mexico to South America, Asia, Africa, and Australia.

9. Rhynchospora Vahl, Enum. Pl. 2: 229. 1805.

\section{Key to species of Rhynchospora of the Araguaia River Basin}

1. Capitulum or corymb.

2. Corymb 9.2. Rhynchospora brevirostris

2'. Capitulum.

3. Leaves not exceeding the inflorescence, achenes biconvex, winged 9.1. R. barbata

3'. Leaves exceeding the inflorescence, achenes trigonous, not winged 9.4. R. globosa

1'. Anthela.

4. Achenes biconvex, ovoid, rugose, tubercles up to $0.5 \mathrm{~mm}$.

4'. Achenes biconcave, oblong to ellipsoid, rugulose or smooth, tubercles 3-3.5 mm.

5. Bristles absent 9.7. R. velutina

5'. Bristles present 9.5. . rugosa

6. Lateral inflorescences absent or 1, tubercles not sulcate 9.6. R. trispicata

6'. Lateral inflorescences 3-4, tubercles sulcate 9.3. R. corymbosa 
9.1 Rhynchospora barbata (Vahl) Kunth, Enum. Pl. 2: 290.1837. Figs. 6a, $8 \mathrm{e}$

Plants amphibious or emergent, $30-60 \mathrm{~cm}$ tall; culms cylindrical, glabrous or pilose. Leaves 3 -ranked, $1-12 \times 0.2-0.3 \mathrm{~cm}$, not exceeding the inflorescence, linear-lanceolate, apex acute, abaxial surface pilose, margins scabrous; sheaths undifferentiated. Capitulum multiflorous, terminal, 1$1.5 \times 0.5-1 \mathrm{~cm}$; external bracts foliaceous. Spikelets 5-8 $\times 0.5-1 \mathrm{~mm}$, spiral, lanceolate; sterile glumes $1-$ $3 \times 1 \mathrm{~mm}$, oval-lanceolate, apex acute, mucronate, cartilaginous; fertile glumes of staminate and bisexual spikelets 4-7 ×0.8-1 mm, lanceolate, apex obtuse, convolute, cartilaginous or membranous. Perianth with 5 bristles, 4-6 $\mathrm{mm}$ long, antrorsely scabrous, base plumose; stamens 3; styles entire; ovaries 2carpellate. Achenes $0.8-1 \times 0.8-1 \mathrm{~mm}$, biconvex, oblong to obovoid, dark brown to black, rugulose, winged; tubercles $1 \times 0.6-0.8 \mathrm{~mm}$, conical.

Material examined: GOIÁS: Aruanã, 29.V.1997, fr., C.P. Bove et al. $218 b$ (R). Caiapônia, 30.IV.1973, fl. and fr., W.R. Anderson 9525 (RB). MATO GROSSO: Nova Xavantina, rio das Mortes, II.1947, fl., D.H. Sick 246 (RB). Nova Xavantina, 6. XII.1967, fl., D. Philcox et al. 3410 (UB). Upper Araguaia River Basin, 10.VI.1966, fl. and fr., H.S Irwin et al. 16877 (R).

Rhynchospora barbata is often mistaken for $R$. globosa. The former is easily recognized by its leaves that do not exceed the inflorescence, its foliaceous bracts and its winged achenes, whereas $R$. globosa has leaves that exceed the inflorescence, oval bracts with mucronate apex and achenes without wings. In the field both species have stamens with long, blue filaments. $R$. barbata is found in rivers and swamps, and occurs in tropical America.

\subsection{Rhynchospora brevirostris Griseb., Cat. Pl. Cub. 246. 1866. \\ Figs. 6b-d, $8 \mathrm{f}$}

Plants amphibious or emergent, $9-14 \mathrm{~cm}$ tall; culms trigonous, glabrous. Leaves $5-14 \times 0.1 \mathrm{~cm}$, 3-ranked and spiral, exceeding the inflorescence, linear, apex acute, trichomes sparse, margins ciliate; sheaths undifferentiated. Corymb multiflorous, $3-$ 4 lateral, 3-4 $\times 2-4 \mathrm{~cm}$; external bracts foliaceous. Spikelets 3-4×0.7-1 mm, fasciculate, ellipsoidal; sterile and fertile glumes of the bisexual spikelets $2-3 \times 0.4-1.5 \mathrm{~cm}$, oval, apex acute to aristate; fertile glumes of the staminate spikelets $2-2.5 \times 0.3-0.5 \mathrm{~mm}$, lanceolate, apex acute. Perianth absent; stamens 2; styles 2-fid; ovaries 2-carpellate. Achenes $0.8-1 \times$ $0.8-1 \mathrm{~mm}$, biconvex, widely obovoid, upper part green, lower part brown, smooth; tubercles $0.1-0.3$ $\times 0.6-0.8 \mathrm{~mm}$, pyramidal.
Material examined: GOIÁS: Piranhas, $85 \mathrm{kmSE}$ of Aragarças, 22.VI.1966, fl., H.S Irwin et al. 17597 (UB). MATO GROSSO: Rio Manso, IV.1911, fl., F.C. Hoehne s.n. (R43568). Xavantina, 13.VI.1966, fl., H.S Irwin et al. s.n. (RB 162141). Barra do Garças, 7.V.1973, fl., W.R. Anderson 9876 (UB). Additional material examined: MATO GROSSO: Campos Novos, III.1916, fl. and fr., T. Boanerges s.n. (R 23479).

Rhynchospora brevirostris is a slender plant with narrow, spreading leaves. It is easily distinguished by its corymb inflorescence and aristate glumes. It is found in rivers. The species occurs from Central to South America, as well as in Africa and Madagascar.

9.3 Rhynchospora corymbosa (L.) Britton, Trans. New York Acad. Sci. 11:84. 1892. Figs. 6e, 8g Plants emergent, $100-150 \mathrm{~cm}$ tall; culms trigonous, angles scabrous. Leaves $40-70 \times 0.5-2 \mathrm{~cm}, 3$-ranked, not exceeding the inflorescence; linear-lanceolate, apex acute, margins and midrib scabrous on the abaxial surface; sheaths undifferentiated. Anthela 3-4 lateral, 5-25 $\times 10-20 \mathrm{~cm}$; external bracts foliaceous. Spikelets 6-7 $\times 1-1.6 \mathrm{~mm}$, fasciculate, obovoid to ellipsoid; sterile glumes 1-4×0.8-2 mm, oval, apex acute, apiculate; fertile glumes of staminate and bisexual spikelets $4-6 \times$ 0.5-1.5 mm oval-lanceolate, apex acute. Perianth with 7 bristles, 4-6 mm long, antrorsely scabrous; stamens 3; styles entire; ovaries 2-carpellate. Achenes 3-3.5 $\times 2$ $2.5 \mathrm{~mm}$, biconvex, obovoid, brown, smooth; tubercles yellowish or light brown, 3-3.5 $\times 1 \mathrm{~mm}$, conical, sulcate. Material examined: GOIÁS: Mozarlândia, 15.XI.1999, fl. and fr., C.P. Bove et al. 614 (R).

Additional material examined: DISTRITO FEDERAL: Brasília 18.XI.1958, fl. and fr., E. Pereira \& G. Pabst 4852 (RB); 18.VIII.1961, fl., Heringer 8591 (RB). GOIÁS: Alto Paraíso, Chapada dos Veadeiros, 9.III.1969, fl., H.S Irwin et al. s.n. (RB 162402). MATO GROSSO: São Félix, córrego do Porco (Porco Stream), 7.V.1968, fl., J.A. Ratter et al. s.n. (RB 176822). Baia Grande, 25.XI.1977, fl. and fr., J.S. Costa s.n. (RB 181438). MATO GROSSO DO SUL: Zuzu, 8.IX.1980, fl. and fr., J.G. Guimarães 1172 (RB); Fazenda Boa Vista, 30.X.1981, fl. and fr., P.P. Furtado s.n. (RB 213432).

Rhynchospora corymbosa is often found in anthropic habitats. Guaglianone (2001) recognized five varieties of $R$. corymbosa, but here only the species is considered. $R$. corymbosa is found in rivers and ponds, and occurs from Central to South America, as well as in Africa, Madagascar, and Asia.

9.4 Rhynchospora globosa (Kunth) Roem. \& Schult., Syst. Veg. 2: 89.1817. Figs. 6f-g, 8h Plants emergent, $70-150 \mathrm{~cm}$ tall; culms trigonous, glabrous. Leaves 50-100 ×0.1-0.3 cm, 3-ranked, exceeding the inflorescence; linear, apex acute, pilose, trichomes black, margins ciliate; sheaths brown, 

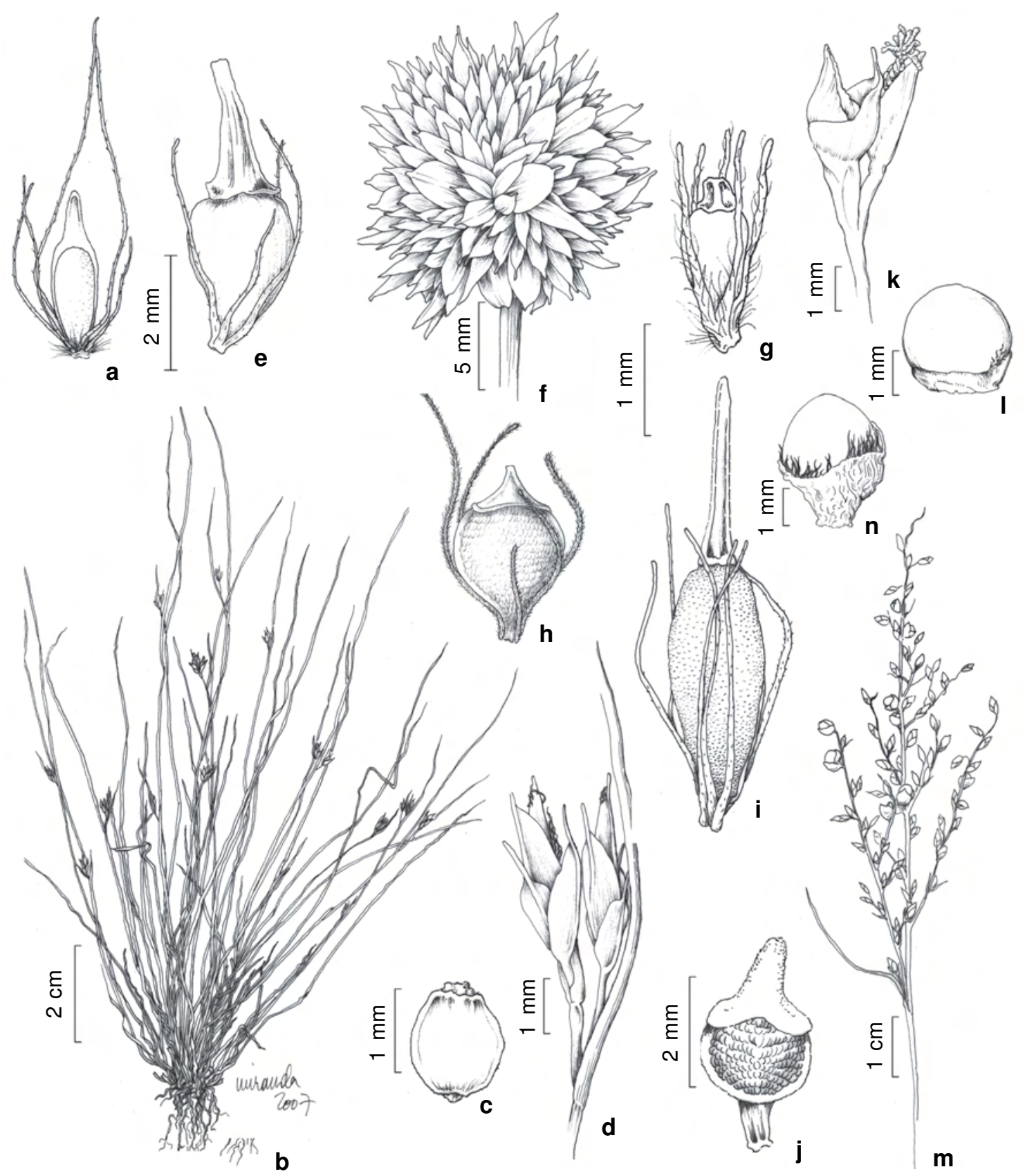

Figure 6 - a. Rhynchospora barbata (Vahl) Kunth - achene (Bove et al. 218b). b-d. R. brevirostris Griseb. - b. habit; c. achene; d. spikelets (Boanerges s.n R 23479). e. R. corymbosa (L.) Britton - achene (Bove et al. 614). f-g. R. globosa (Kunth) Roem. \& Schult. - f. capitulum of spikelets; g. achene (G.T. Prance et al. s.n. R 205749). h. R. rugosa (Vahl) Gale - achene (D.R. Hunt 5906). i. R. trispicata (Nees) Schrad. ex Steud - achene (Bove et al. 1658). j. R. velutina (Kunth) Boeck - achene (Rizzo 6106). k-1. Scleria melaleuca Rchb. ex Schltdl. \& Cham. - k. staminate and pistillate spikelets; 1. achene (Bove et al.1653). m-n. S. mitis P. J. Bergius - m. detail of a panicle of spikelets. n. achene (Bove et al. 564). 
expanded. Capitulum multiflorous, terminal, $1-2 \times 1-$ $2 \mathrm{~cm}$; external bracts glumaceous. Spikelets 5-7 $\times 1-$ $3 \mathrm{~mm}$, spiral, lanceolate; sterile glumes $3-5 \times 1-1.5 \mathrm{~mm}$, oval, apex mucronate; fertile glumes of staminate and bisexual spikelets 3-6×0.1-0.3 mm, lanceolate, apex acute, mucronate. Perianth with 5 bristles, ca. $2 \mathrm{~mm}$ long, plumose, antrorsely scabrous on the upper third; stamens 3; styles 3-fid; ovaries 3-carpellate. Achenes 1.5-2 $\times 0.5-1 \mathrm{~mm}$, trigonous, obovoid, light brown, smooth, costate at the angles, costa pilose; tubercles $0.3-0.4 \times 0.4-0.5 \mathrm{~mm}$, rectangular.

Material examined: GOIÁS: Caiapônia, 2.V.1973, fl., W.R. Anderson 9652 (R, UB). MATO GROSSO: Nova Xavantina, 8.VI.1968, fl., R.R. Santos et al. s.n. (RB 176824).

Additional material examined: State of Mato Grosso, Brazil: sl., road between Buriti and Água Fria, 23.X.1973, fl. and fr., G.T. Prance et al. s.n. (R 205749).

See comments for Rhynchospora barbata.

R. globosa occurs from Central America to tropical South America.

9.5 Rhynchospora rugosa (Vahl) Gale, Rhodora 46: 275. 1944.

Figs. 6h, 8i

Plant amphibious or emergent, $27-200 \mathrm{~cm}$ tall; culms trigonous, glabrous. Leaves $5-55 \times 0.1 \mathrm{~cm}, 3-$ ranked, not exceeding the inflorescence; linear, apex acute, glabrous; sheaths undifferentiated. Anthela 0-3 lateral, 1-5 × 0.9-1.6 cm; external bracts foliaceous. Spikelets 3-6 mm, fasciculate, ovallanceolate; sterile and fertile glumes of the bisexual spikelets 2-4×1-1.3 mm, oval, oval-lanceolate, apex acute or mucronate; fertile glumes of the staminate spikelets 5-7 $\times 1 \mathrm{~mm}$, lanceolate, apex acute. Perianth with $7-8$ bristles, $4-7 \mathrm{~mm}$ long, antrorsely scabrous; stamens 1; styles 2-fid; ovaries 2carpellate. Achenes 2-2.5 × 1-1.3 mm, biconvex, ovoid, brown, rugose; tubercles $0.5-0.7 \times 0.5-0.8$ $\mathrm{mm}$, conical.

Material examined: GOIÁS: Caiapônia, 29.VI.1966, fl. and fr., H.S Irwin et al.17969 (UB). MATO GROSSO: Nova Xavantina, 10.VI.1966, fl. and fr., D.R. Hunt 5906 (RB); $14^{\circ} 38^{\prime} \mathrm{S} 52^{\circ} 14^{\prime} \mathrm{W}, 15 . \mathrm{VII} .1968$, fl. and fr., G.C. Argent \& P.W. Richards 6443 (RB).

Rhynchospora rugosa is similar to $R$. velutina, and in the vegetative state it is very difficult to separate the two species. The only way to avoid misidentification is through the observation of the flower or the achene. The former species has flowers and achenes with 7-8 bristles, whereas $R$. velutina has no bristles. $R$. rugosa occurs from Central to South America, as well as in Africa, Madagascar, and Asia.
9.6 Rhynchospora trispicata (Nees) Schrad. ex Steud., Syn. Pl. Glumac. 2: 145.1855. Figs. 6i, 9a Plants emergent, $100-130 \mathrm{~cm}$ tall; culms trigonous, glabrous. Leaves $40-70 \times 0.5-1 \mathrm{~cm}, 3-$ ranked, not exceeding the inflorescence; linear, apex acute, glabrous; sheaths undifferentiated. Anthela, 0-1 lateral, 20-30 $\times 20-30 \mathrm{~cm}$; external bracts foliaceous. Spikelets $5-8 \mathrm{~mm}$, fasciculate, ellipsoid; sterile and fertile glumes of bisexual spikelets 2-3 $\times 1-2 \mathrm{~mm}$, oval, apex acute or mucronate; fertile glumes of staminate spikelets 5-7 $\times 1 \mathrm{~mm}$, lanceolate, apex acute. Perianth with 6 bristles, 4-7 mm long, antrorsely scabrous; stamens 3; styles entire; ovaries 2-carpellate. Achenes $4 \times 2 \mathrm{~mm}$, biconcave, oblong to ellipsoid, greenish brown, rugulose; tubercles 3-3.5 $\times 0.2$ $0.4 \mathrm{~mm}$, conical.

Material examined: GOIÁS: Nova Crixás, 14.IV.2006, fl. and fr., C.P. Bove et al. 1658 (R).

Additional material examined: MATO GROSSO: Poconé, 28.VII.1977, fl. and fr., A. Allem \& Vieira 1040 (RB); 153'ㅇ' 6007’W, 1985, J.S Costa 1249 (RB).

Rhynchospora trispicata is easily distinguished by anthelas in multiples of three, with a central sessile anthela or short rachis. It is found in temporary ponds, and occurs in tropical America.

9.7 Rhynchospora velutina (Kunth) Boeck., Vidensk. Meddel. Dansk Naturhist. Foren. Kjobenhavn 149: $1869 . \quad$ Figs. 6j, 9b

Plant emergent, $50-120 \mathrm{~cm}$ tall; culms trigonous, glabrous or velutinous. Leaves 27$65 \times 0.3-2 \mathrm{~cm}, 3-$ ranked and spiral, not exceeding the inflorescence; linear-lanceolate, apex acute, velutinous or pilose; sheaths undifferentiated. Anthela, 0-1 lateral, 14-16× 4-5 cm; external bracts foliaceous. Spikelets 4$5 \times 1-1.3 \mathrm{~mm}$, fasciculate, lanceolate; sterile glumes 2-4 × 1-2 mm, oval, apex mucronate; fertile glumes of staminate and bisexual flowers 2-5 × 0.8-1 mm, oval, oval-lanceolate, apex acute, mucronate. Perianth absent; stamens 3; styles 2-fid; ovaries 2-carpellate. Achenes 1.5$2 \times 1.8 \mathrm{~mm}$, biconvex, ovoid, brown, rugose; tubercles $0.5 \times 0.2-0.3 \mathrm{~mm}$, conical.

Material examined: GOIÁS: Amorinópolis, 20.II.1971, fl. and fr., J.A. Rizzo 6106 (UFG). MATO GROSSO: Alto Araguaia, Córrego do Sapo, 14.I.2004, fl. and fr., C.P. Bove et al. 1327 (R). Xavantina, 31.V.1966, fl. and fr., H.S Irwin et al. 16328 (UB).

See comments for Rhynchospora rugosa. $R$. velutina is found in swamps and temporary ponds. It occurs in tropical America. 
10. Scleria P.J. Bergius, Kongl. Vetensk. Acad. Handl. 26: 142, pl. 4-5. 1765.

\section{Key to species of Scleria of the Araguaia River Basin}

1. Hypogynium 3-lobed, leaves without winged sheaths 10.1. Scleria melaleuca

1'. Hypogynium collar-like, leaves with winged sheaths 10.2. S. mitis

10.1 Scleria melaleuca Rchb. ex Schltdl. \& Cham., Linnaea 6: 29. 1831.

Figs. 6k-1, 9c-d

Plants amphibious or emergent, $50-70 \mathrm{~cm}$ tall. Leaves $15-30 \times 0.5-0.7 \mathrm{~cm}$; linear, apex acute, glabrous, margins ciliate; sheaths not winged, apex acute. Peduncles $0.5-6 \mathrm{~cm}$ long. Panicles multiflorous 2-3, lateral, $2-7 \mathrm{~cm}$ long. Staminate spikelets 3-7 $\times 1.2-1.2-2 \mathrm{~mm}$; lanceolate, peduncle 1-2 mm long; sterile glumes of staminate spikelets 2-3 $\times 1 \mathrm{~mm}$, oblong, apex acute, glabrous; fertile glumes of staminate spikelets $2-4 \times 0.8-1 \mathrm{~mm}$, oval, apex mucronate, convolute, glabrous. Stamens 1. Pistillate spikelets $3-4 \times 1.2-1.8 \mathrm{~mm}$, ovoid to obovoid, sessile; sterile glumes of pistillate spikelets 3-4 $\times 0.8-1.5 \mathrm{~mm}$, oval, oval-lanceolate, apex mucronate, glabrous or ciliate margins; fertile glumes of pistillate spikelets $3-4 \times 2-2.5 \mathrm{~mm}$, oval-orbicular, apex acuminate to mucronate, midrib pilose on the adaxial surface, margins ciliate. Achenes $2 \times 2 \mathrm{~mm}$, white to yellowish, opaque, pilose; hypogynium 3lobed, brown, glabrous.

Material examined: GOIÁS: Aruanã, 14\%49'10"S, $50^{\circ} 58^{\prime} 36.4^{\prime \prime} \mathrm{W}, 13 . \mathrm{IV} .2006$, fl. and fr., C.P. Bove et al. 1653 (R). TOCANTINS: Araguaína, Rio das Lontras, 13.III.1968, fl. and fr., H.S Irwin et al. s.n. (RB 161811).

Scleria melaleuca in vegetative state is similar to $S$. mitis. The former has no winged leaf sheaths and a 3-lobed hypogynum, whereas $S$. mitis has winged leaf sheaths and a collar-like hypogynium. $S$. melaleuca is found in rivers, swamps and temporary ponds, and occurs from Central to South America, as well as in tropical Africa, and Madagascar.

10.2 Scleria mitis P.J. Bergius, Kongl. Vetensk. Acad. Handl. 26: 145, pl. 5. 1765 . Figs. 6m-n, 9e-f

Plants amphibious or emergent, $170-200 \mathrm{~cm}$ tall. Leaves $30-42 \times 1-1.5 \mathrm{~cm}$; linear or lanceolate, pilose, margins scabrous; sheaths winged, apex acuminate. Peduncles 1-12 cm long. Panicles multiflorous 4-5, lateral, 9-20 cm long. Staminate spikelets 3-4×1-1.5 mm, ellipsoid, peduncle 1-3 $\mathrm{mm}$ long; sterile glumes of the staminate spikelets 2-2.3 $\times 1-1.8 \mathrm{~mm}$, oval, apex mucronate, adaxial surface pilose in the midrib, margins ciliate; fertile glumes of the staminate spikelets $2-2.5 \times 0.5-0.8 \mathrm{~mm}$, oblong, apex acute to obtuse, glabrous. Stamens 3. Pistillate spikelets $2-3 \times 1-3 \mathrm{~mm}$, ovoid to orbiculate, peduncle absent to $0.5 \mathrm{~mm}$; sterile and fertile glumes of the pistillate spikelets similar, $2-3 \times 1-2 \mathrm{~mm}$, ovalorbicular, mucronate, concave, glabrous. Achenes $2.5-3.5 \times 2.5-3 \mathrm{~mm}$, white, shiny, glabrous; hypogynium collar-like, greenish brown, margins pilose, trichomes red.

Material examined: GOIÁS: Jussara, 12.XI.1999, fl. and fr., C.P. Bove et al. 564 (R).

Additional material examined: GOIÁS: Rio Verde, 19.IV.1978, fl. and fr., G.J. Sheperd et al. 7452 (RB). MATO GROSSO: Aquidauana, 1949, D.A. Correia s.n. (RB 81371). Maracaju, 29.XII.1973, fl. and fr., D. Sucre 410494 (RB).

See comments for Scleria melaleuca. S. mitis is found in buriti palm swamps (Mauritia flexuosa); it occurs from Central to South America.

\section{Discussion}

The present floristic survey does not claim to be the last word on Cyperaceae diversity in the Araguaia River Basin, but it aims to present available knowledge after intensive field and herbarium work. Efforts to access the diversity of aquatic ecosystems through scientific collections were hindered by the lack of information on specimen labels about the environment and seasonal characteristics of most plants. An in situ research is the best route to a good overview. In spite of being very common in the Brazilian Cerrado with about 200 species (Sano et al. 2008), the Cyperaceae is present in a single study published on the flora of the Grão Mongol region, state of Minas Gerais (Vitta \& Prata 2009). This paper describes 37 species, 15 of them hydrophytic. Although both the Araguaia River Basin and the Grão Mongol Mountain Range are located within the Brazilian Cerrado, these regions are ecologically different. There are 23 species described in the present study that were not found in the flora of Grão Mogol. Eight other species (Ascolepis brasiliensis, Cyperus haspan, C. unioloides, Eleocharis capillacea, E. filiculmis, Fuirena umbellata, Rhynchospora globosa and $R$. rugosa) are common to both areas. 

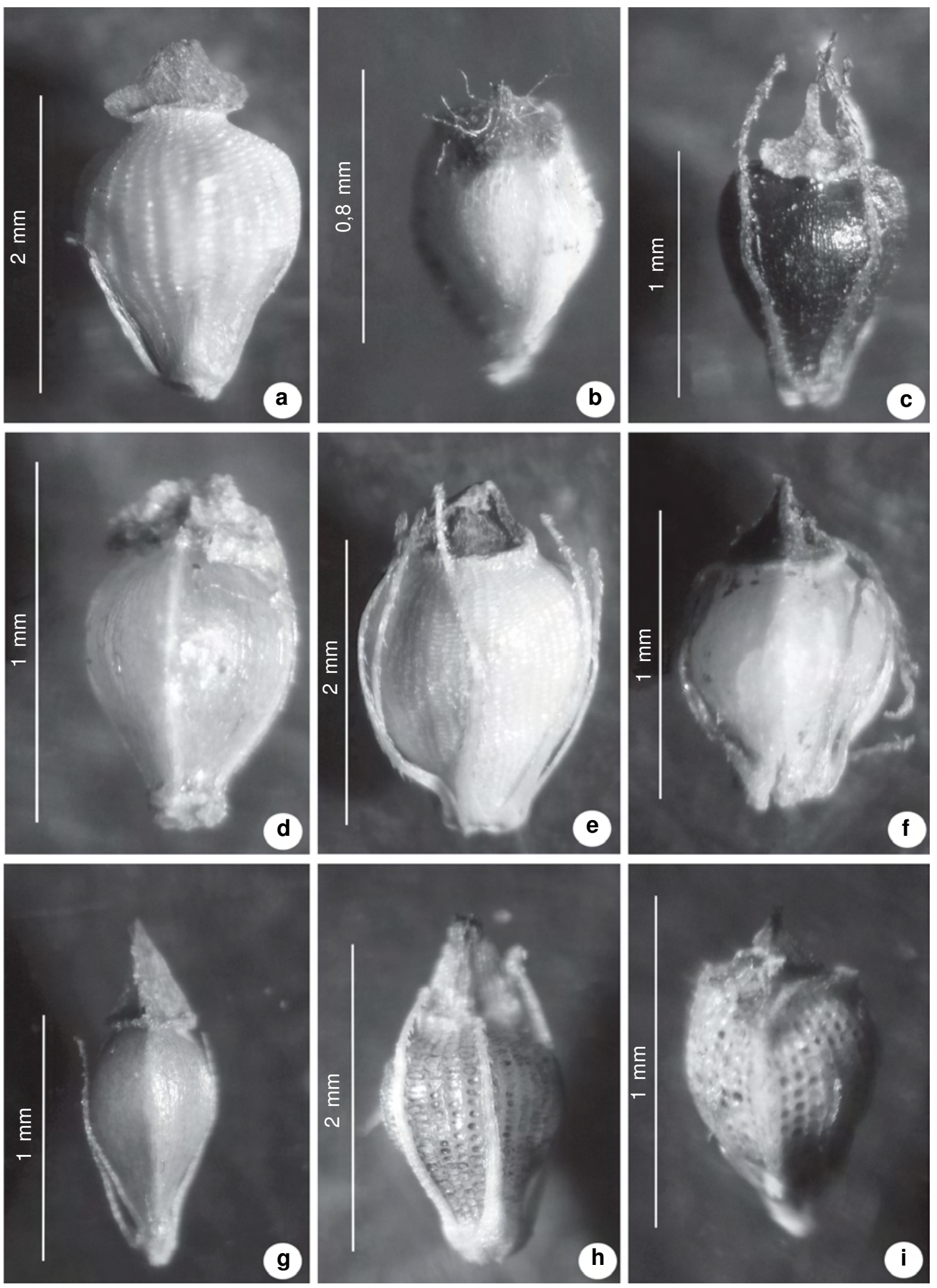

Figure 7 - a-i. Achene. a. Eleocharis acutangula (Roxb.) Schult. (Bove et al. 290). b. E. bicolor Chapm. (Bove et al. 592). c. E. capillacea Kunth (Bove et al. 1153). d. E. filiculmis Kunth (Bove et al. 184). e. E. interstincta (Vahl) Roem. \& Schult. (Bove et al. 552). f. E. nana Kunth (Bove et al. 759). g. E. nudipes (Kunth) Palla (Bove et al. 185). h. E. plicarhachis (Griseb.) Schult. (Bove et al. 230b). i. E. retroflexa (Poir.) Urb. (Bove et al. 1649). 

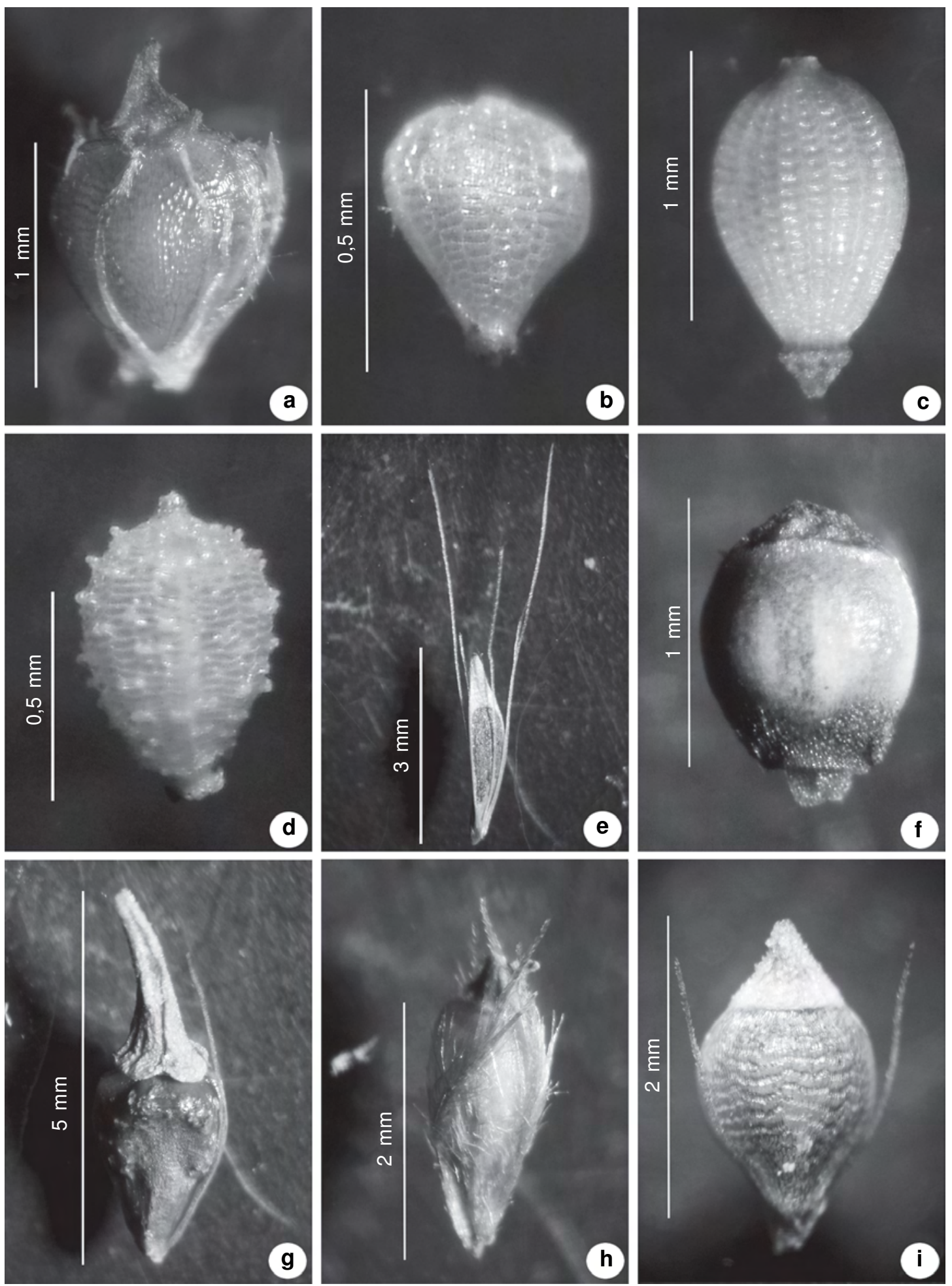

Figure 8 - a-i. Achene. a. Eleocharis sellowiana Kunth (Bove et al. 1326). b. Fimbristylis aestivalis (Retz.) Vahl (Bove et al. 608a). c. F. dichotoma (L.) Vahl (Bove et al. 510). d. F. miliacea (L.) Vahl (Bove et al. 1668). e. Rhynchospora barbata (Vahl) Kunth (Bove et al. 218b). f. Rhynchospora brevirostris Griseb. (Boanerges s.n. R 23479). g. R. corymbosa (L.) Britton (Bove et al. 614). h. R. globosa (Kunth) Roem. \& Schult. (G.T. Prance et al. s.n. R 205749). i. R. rugosa (Vahl) Gale (D.R. Hunt 5906). 

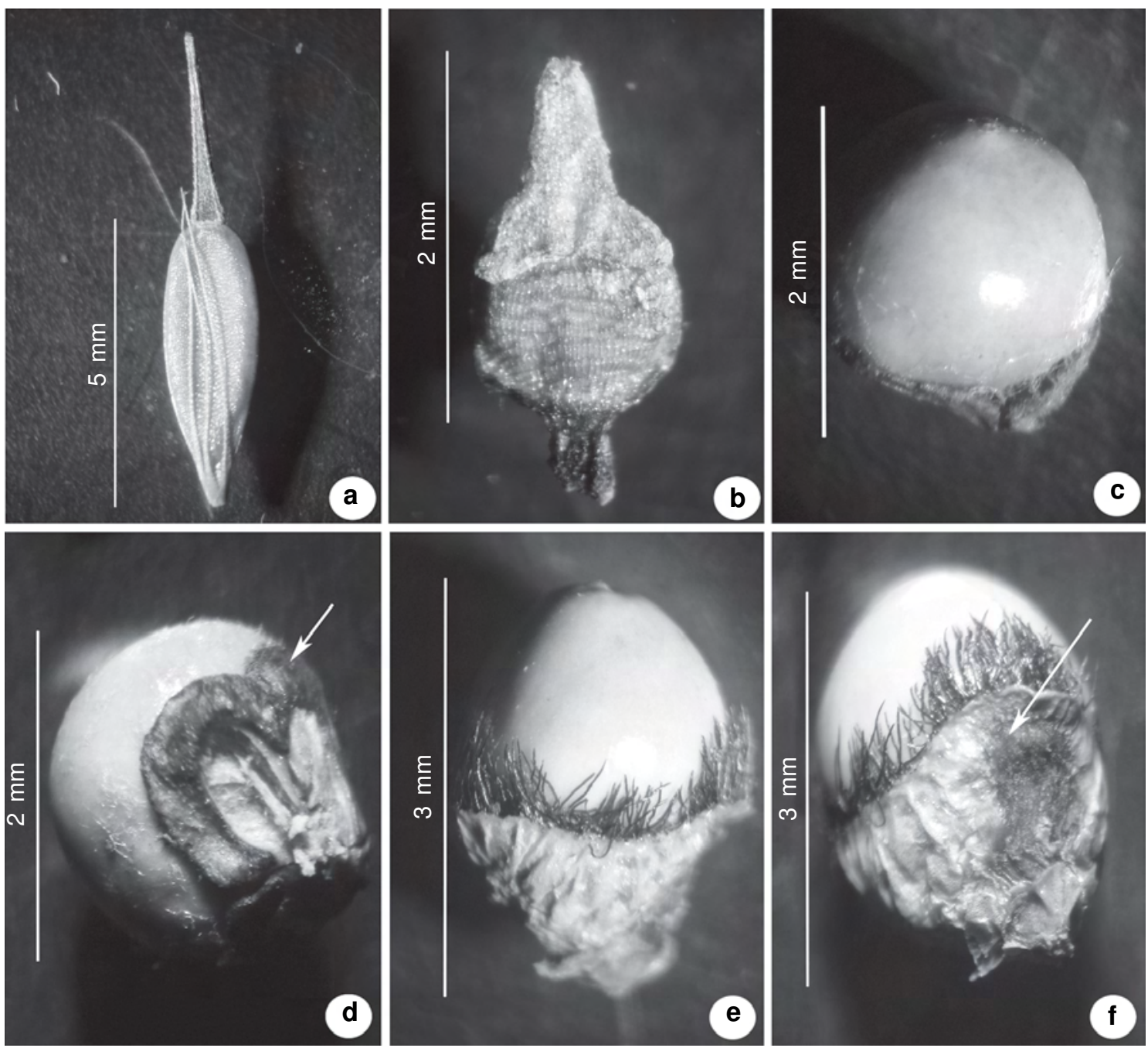

Figure 9 - a-f. Achene. a. R. trispicata (Nees) Schrad. ex Steud. (Bove et al. 1658). b. R. velutina (Kunth) Boeck. (Bove et al. 1327). c-d Scleria melaleuca Rchb. ex Schltdl. \& Cham. - d. detail showing a 3-lobed hypogynium (arrow) (Bove et al. 1653). e-f. Scleria mitis P. J. Bergius - f. detail showing a collar-like hypogynium (arrow) (Bove et al. 564).

Several checklists for the Cerrado include Cyperaceae species, but few of them include specimens collected in the Araguaia River Basin (Dubs 1998; Mendonça et al. 1998; Batalha \& Martins 2002; Sano et al. 2008; Gil et al. 2007). Dubs (1998) listed 133 Cyperaceae species for the states of Mato Grosso and Mato Grosso do Sul, and five of them occur also in the Araguaia River Basin, but only Rhynchospora globosa (Kunth) Roem. \& Schult. was found in aquatic environments. Batalha \& Martins (2002) listed 12 species for the Emas National Park, located in the Araguaia River Basin, but only R. rugosa (Vahl) Gale was found in aquatic environments.

Life-form categories represent different degrees of adaptation to aquatic life and are highly convergent among aquatic angiosperms (Philbrick \& Les 1996). The emergent form is predominant among the hydrophytic Cyperaceae of the Araguaia River Basin. Twenty-one species are exclusively emergent (67.7\%), eight species are emergent or amphibious (25.8\%), and two species are emergent or submerged (6.5\%). The predominance of emergent forms can be explained by the presence of several temporary flooded areas (seasonal aquatic environments) and marginal areas of permanent aquatic ecosystems.

Despite the large area of the Araguaia River Basin and the high diversity of wetland habitats within the Brazilian Cerrado, no species presented in this study were mentioned on the official Brazilian red list (MMA 2008). However, few studies on the hydrophytic 
flora of this region have been published (Koehler \& Bove 2001; 2004; Gil et al. 2007; Philbrick et al. 2010). Such studies are becoming increasingly important, as human impacts on wetland regions and their flora intensify, and the need for conservation measures becomes more urgent. Historically, a high diversity of wetland habitats was associated with the Araguaia River (Gil et al. 2007). Since the 1970s, many projects have led to reduction in both diversity and area of wetlands. The construction of the Belém-Brasília Highway and hydroelectric dams, as well as the expansion of agricultural and mining activities (Moss $\&$ Moss 2005), caused major impacts on those areas.

\section{Acknowledgments}

We thank the curators of the herbaria R, RB, UB and UFG. Wilson Costa and Josele Paz helped in the field, Claudia Miranda provided us with illustrations, Gustavo Gritz provided us with photomicrographs, Wilson Costa gave invaluable suggestions on the manuscript, and Janet Reid made corrections on the English version. We also would like to thank the anonymous reviewers for their precious suggestions.

\section{References}

Adams, C.D. 1994. Cyperaceae. In: Davidse, G.M.; Souza, S. \& Chater, O. (eds.). Flora mesoamericana. Vol. 6. Universidad Nacional Autónoma de México, Ciudad de México. Pp. 402-485.

Alves, M.; Araújo, A.C.; Hefler, S.M.; Trevisan, R.; Silveira, G.H. 2010. Cyperaceae. In: Forzza, R.C. et al. (eds.). Catálogo de plantas e fungos do Brasil. Vol. 2. Andrea Jakobsson Estúdio; Instituto de Pesquisas Jardim Botânico do Rio de Janeiro, Rio de Janeiro. Pp 903-922.

Alves, M.; Araújo, A.C.; Prata, A.P.; Vitta, F.; Hefler, S.; Trevisan, R.; Gil, A.S.B.; Martins, S \& Thomas, W. 2009. Diversity of Cyperaceae in Brazil. Rodriguésia 60: 771-782.

Batalha, M.A. \& Martins, F.R. 2002. The vascular flora of the cerrado in Emas National Park (Goiás, Central Brazil). Sida 20: 295-311.

Cook, C.D.K. 1996. Aquatic plant book. $2^{\mathrm{a} e d .}$ SPB Academic Publishing, Amsterdam. 228p.

Dubs, B. 1998. Prodromus Florae Matogrossensis: The botany of Mato Grosso. Betrona-Verlag, Küsnacht. 444p.

Gil, A.S.B.; Oliveira, A.L.R. \& Bove, C.P. 2007. Listagem florística das Cyperaceae hidrófilas da região do Alto e Médio rio Araguaia, Goiás, Mato Grosso e Tocantins, Brasil - Parte 1. Revista de Biologia Neotropical 4: 101-110.

Goetghebeur, P. 1998. Cyperaceae. In: Kubitzki, K. (ed.). The families and genera of vascular plant: flowering plants-monocotyledons. Vol. 4. Springer-Verlag, Berlin. Pp. 141-190.

Govaerts, R.; Simpson, D.A.; Bruhl, J.; Egorova, T.; Goetghebeur, P. \& Wilson, K. 2007. World checklist of Cyperaceae - Sedges. Kew Gardens Publishing, Kew. 765p.

Guaglianone, E.R. 2001. Contribution to the study of the genus Rhynchospora (Cyperaceae) V. Section Longirostres in Austral America. Darwiniana 39: 287-342.

Koehler, S. \& Bove, C.P. 2001. Hydrocharitaceae from central Brazil: a new species of Egeria and a note on Apalanthe granatensis. Novon 11: 63-66.

Koehler, S. \& Bove, C.P. 2004. Alismatales from the upper and middle Araguaia river basin (Brazil). Revista Brasileira de Botânica 27: 439-452.

Lima, J.E.F.W.; Santos, P.M.C.; Carvalho, N.O. \& Silva, E.M. 2004. Diagnóstico do fluxo de sedimentos em suspensão na Bacia Araguaia-Tocantins. Embrapa Cerrados, Planaltina; ANEEL/ANA, Brasília. 117p.

MMA - Ministério do Meio Ambiente. 2008. Lista oficial das espécies da flora brasileira ameaçadas de extinção. Intrução Normativa de setembro de 2008, Brasília, Brasil, Anexo 1. Pp. 3-55.

Mendonça, R.C.; Felfili, J.M.; Walter, B.M.T.; Júnior, M.C.S.; Rezende, A.V.; Filgueiras, T.S. \& Nogueira, P.E. 1998. Flora vascular do Cerrado. In: Sano, S.M. \& Almeida, S.P. (eds.). Cerrado: ambiente e flora. EMBRAPA-CPAC, Brasília. Pp. 289-556.

Moss, G. \& Moss, M. 2005. Brasil das águas, revelando o azul do verde e amarelo. Supernova, São Paulo. 158p.

Myers, N; Mittermeier, R.A.; Mittermeier, C.G.; Fonseca, G.A.B. \& Kent, J. 2000. Biodiversity hotspots for conservation priorities. Nature 403: 853-858.

Pedralli, G. 1988. Macrófitos aquáticos: as plantas fiscais. Ciência Hoje 7: 76.

Philbrick, C.T.; Bove, C.P. \& Stevens, H.I. 2010. Endemism in neotropical Podostemaceae. Annals of the Missouri Botanical Garden 97: 425-456.

Philbrick, C.T. \& Les, D.H. 1996. Evolution of aquatic angiosperm reproductive systems. BioScience 46: 813-826.

Resende, M.; Curi, N.; Resende, S.B. \& Correia, G.F. 1995. Pedologia: bases para distinção de ambientes. NEPUT, Viçosa. 304p.

Sano, S.M.; Almeida, S.P. \& Ribeiro, J.F. 2008. Cerrado: ecologia e flora. Vol. 2. EMBRAPA-CPAC, Planaltina. 876p.

Thiers, B. [2010 and continuously updated]. Index Herbariorum: a global directory of public herbaria and associated staff. New York Botanical Garden's Virtual Herbarium. Disponível em <http:// sweetgum.nybg.org/ih/>.

Vitta, F.A. \& Prata, A.P. 2009. Flora de Grão-Mogol: Cyperaceae. Boletim de Botânica da Universidade de São Paulo 27: 43-62. 